\title{
Symmetric tops in combined electric fields: Conditional quasisolvability via the quantum Hamilton-Jacobi theory
}

\author{
Konrad Schatz and Bretislav Friedrich* \\ Fritz-Haber-Institut der Max-Planck-Gesellschaft, Faradayweg 4-6, D-14195 Berlin, Germany \\ Simon Becker and Burkhard Schmidt ${ }^{\dagger}$ \\ Institute for Mathematics, Freie Universität Berlin, Arnimallee 6, D-14195 Berlin, Germany
}

(Received 23 February 2018; published 31 May 2018)

\begin{abstract}
We make use of the quantum Hamilton-Jacobi (QHJ) theory to investigate conditional quasisolvability of the quantum symmetric top subject to combined electric fields (symmetric top pendulum). We derive the conditions of quasisolvability of the time-independent Schrödinger equation as well as the corresponding finite sets of exact analytic solutions. We do so for this prototypical trigonometric system as well as for its anti-isospectral hyperbolic counterpart. An examination of the algebraic and numerical spectra of these two systems reveals mutually closely related patterns. The QHJ approach allows us to retrieve the closed-form solutions for the spherical and planar pendula and the Razavy system that had been obtained in our earlier work via supersymmetric quantum mechanics as well as to find a cornucopia of additional exact analytic solutions.
\end{abstract}

DOI: 10.1103/PhysRevA.97.053417

\section{INTRODUCTION}

A realization of a quantum symmetric top is a molecule that possesses at least a threefold rotational symmetry axis. The symmetric top rotational states, $|J, K, M\rangle$, are characterized by the angular momentum quantum number, $J$, and the projections, $K$ and $M$, of the angular momentum on the body- and space-fixed axis, respectively. Polar symmetric top molecules, i.e., those with a body-fixed electric dipole moment, exhibit a unique behavior in electric fields: In their precessing states, in which $J, K$, and $M$ are all nonzero, the body-fixed electric dipole does not average out in first order, as a result of which such states are inherently oriented in the field [1,2]. Like other polar molecules, symmetric tops are also amenable to pendular orientation, a higher-order effect that involves hybridization over both even and odd $J$ 's of the top's pure rotational states. Pendular states of a different kind-that exhibit alignment rather than orientation - can be created via the induced-dipole interaction of an external electric or optical field with the anisotropic polarizability of the symmetric top; in this case, the rotational hybrids comprise either even or odd $J$ 's. We note that orientation is like a single-headed arrow pointing in a certain direction whereas alignment is like a double-headed arrow directed along a certain direction. A combination of the permanent and induced-dipole interactions provides versatile means to orient symmetric top molecules, often with only a small admixture of the parity-breaking orienting permanent dipole interaction to the parity-conserving aligning induceddipole interaction $[2,3]$.

At the same time, a symmetric top molecule under the combined permanent and induced dipole interaction represents

\footnotetext{
*bretislav.friedrich@fhi-berlin.mpg.de

†burkhard.schmidt@fu-berlin.de
}

a realization of a symmetric top quantum pendulum [2]. As such it is a prototypical quantum system that lurks behind numerous applications, some of which are summarized in Table I. Unlike other key quantum prototypes, such as the harmonic oscillator, the quantum pendulum lacks, in general, exact eigenproperties, and so its energies and wave functions have to be obtained by solving the corresponding Schrödinger equation numerically [33]. However, as exemplified in our previous work on the three-dimensional (3D) quantum pendulum, realized by a polar and polarizable ${ }^{1} \Sigma$ molecule (a special case of a symmetric top with $K=0),{ }^{1}$ there are classes of eigenproblems in quantum mechanics that are neither exactly solvable nor unsolvable, but lie somewhere in between, i.e., possess a finite number of exact solutions for specific values of the interaction or other defining parameters (see Table II). The analytic, closed-form, and algebraic solutions as defined in Table II and presented below are always exact solutions, i.e., no approximation is involved in obtaining them.

Herein, we explore quasisolvability of the symmetric top quantum pendulum within the framework of the quantum Hamilton-Jacobi $(\mathrm{QHJ})$ theory $[57,58]$ and find the conditions for its quasisolvability as well as the closed-form solutions themselves. In the process, we retrieve the conditions of quasisolvability and the closed-form solutions derived previously for the planar (two-dimensional) and spherical (3D) quantum pendulum within the framework of supersymmetric quantum mechanics (SUSY QM) [32,33,59-61] as well as identify a cornucopia of further closed-form solutions and the conditions under which they obtain.

\footnotetext{
${ }^{1}$ We note that linear molecules in states with nonzero electronic angular momentum that fall under Hund's cases (a) and (c) are genuine symmetric tops, with $K \neq 0$ [52].
} 
TABLE I. Examples of problems and applications in chemistry and physics where the trigonometric symmetric top pendulum and its special cases make a prominent appearance. See also Ref. [4]. We note that the hyperbolic symmetric top potential resembles the generalized Pöschl-Teller potential [5].

\begin{tabular}{lc}
\hline \hline Problems and applications & Reference \\
\hline Molecular alignment/orientation & {$[6-15]$} \\
Deflection, focusing, trapping & {$[16-20]$} \\
Reaction stereodynamics & {$[21-23]$} \\
Stark spectroscopy & {$[24-26]$} \\
Molecules in combined fields & {$[27-33]$} \\
Photoelectron angular distributions & {$[34-36]$} \\
Diffraction from within & {$[37]$} \\
High-order harmonic generation and orbital imaging & {$[38-42]$} \\
Quantum simulation and computing & {$[43-51]$} \\
\hline \hline
\end{tabular}

In the case of SUSY QM, the closed-form solutions follow from a suitable ansatz for the superpotential, which requires an "educated guess" that is quite hard to make. In contrast, the QHJ theory offers a generic way of constructing such solutions, but calls for an educated guess concerning the choice of appropriate coordinates in which to express them; this is an easier task than coming up with an ansatz for the superpotential.

Moreover, we show that both the exact (in fact, algebraic; see Table II) and numerical spectra of the symmetric top pendulum exhibit patterns that are intrinsically related to those of the eigenproblem obtained by the anti-isospectral transformation $[61,62]$ that converts the trigonometric symmetric top to a hyperbolic one.

The present paper is structured as follows. In Sec. II, we lay out the Hamiltonians of the trigonometric as well as hyperbolic symmetric tops and the corresponding Schrödinger equations the quasisolvability of which we investigate. In Sec. III, we derive for either top the QHJ equation which is solved by the quantum momentum function (QMF). The quantum momentum function is closely related to the SUSY QM superpotential which is, in turn, linked to the ground-state wave funtion. We then derive the conditions of quasisolvability by analyzing the singularity structure of the tops' potential and subsequently construct the excited-state solutions algebraically. The physical relevance of the closed-form solutions found is evaluated by making use of the limit-point and limitcircle classifications. In Sec. IV, we provide a sampling of the closed-form solutions obtained from the QHJ theory for the trigonometric and hyperbolic symmetric top as well as derive their special cases, the planar and spherical pendula and the Razavy system.

\section{SYMMETRIC TOP HAMILTONIAN}

The Hamiltonian of a symmetric top molecule subject to collinear electric fields with interaction strengths $\eta$ and $\zeta$ is given by

$$
\mathcal{H}_{t}=B \mathbf{J}^{2}-B \rho J_{3}^{2}+\mathcal{V}_{t}(\theta)
$$

where

$$
\mathcal{V}_{t}(\theta)=-\eta \cos \theta-\zeta \cos ^{2} \theta
$$

is the potential. The trigonometric character of the Hamiltonian and its potential is emphasized by the subscript $t$. The interaction strengths $\eta$ and $\zeta$ arise, respectively, from the fields $\varepsilon_{1}$ and $\varepsilon_{2}$ such that

$$
\eta=\mu \varepsilon_{1} \quad \text { and } \quad \zeta=\zeta_{\|}-\zeta_{\perp} \quad \text { with } \quad \zeta_{\|, \perp}=\frac{\alpha_{\|, \perp} \varepsilon_{2}^{2}}{2}
$$

where $\mu$ and $\alpha_{\|, \perp}$ denote the body-fixed electric dipole moment and principal polarizability components parallel and perpendicular to the body-fixed axis 3 (the figure axis) [2] and the parameter $\rho$ determines whether the inertia tensor of the symmetric top is prolate or oblate:

$$
\rho=\left\{\begin{array}{ll}
A / B-1>0 & \text { prolate } \\
C / B-1<0 & \text { oblate }
\end{array} .\right.
$$

Here $A=\frac{\hbar^{2}}{2 I_{A}}, B=\frac{\hbar^{2}}{2 I_{B}}$, and $C=\frac{\hbar^{2}}{2 I_{C}}$ are the rotational constants defined via the principal moments of inertia $I_{A}, I_{B}$, and $I_{C}$.

In terms of the Euler angles $(\varphi, \theta, \chi)$, the body-fixed components $(1,2,3)$ of the angular momentum operator, $\mathbf{J}$, are given by

$$
\begin{gathered}
J_{1}=i\left(-\sin \chi \partial_{\theta}+\frac{\cos \chi}{\sin \theta} \partial_{\varphi}-\cot \theta \cos \chi \partial_{\chi}\right), \\
J_{2}=i\left(-\cos \chi \partial_{\theta}-\frac{\sin \chi}{\sin \theta} \partial_{\varphi}+\cot \theta \sin \chi \partial_{\chi}\right), \\
J_{3}=-i \partial_{\chi}
\end{gathered}
$$

TABLE II. Classification of quantum systems according to their solvability [53]. If the solutions within a solvability class for a given system are only obtained under certain conditions imposed on the system's parameters, the system is termed conditionally QS, QES, or QPS [54,55]. Furthermore, we use the following terminology: An analytic solution is a solution in terms of elementary functions (the set of elementary functions is not closed under limits and infinite sums) and of (some) special functions (except for those that are infinite sums); a closed-form solution is an analytic solution obtained via a finite number of operations (a narrower class than analytic solutions that in practice excludes some special functions); an algebraic solution is a closed-form solution built up from integer constants and algebraic operations (addition, multiplication, exponentiation). A comprehensive overview is given in Ref. [56].

\begin{tabular}{lccc}
\hline \hline Class & Spectrum & Normalizability & Number of solutions \\
\hline Exactly solvable, ES & Entire & Normalizable & Infinite \\
Quasisolvable, QS & Part & Normalizable and non-normalizable & Finite \\
Quasiexactly solvable, QES & Part & Normalizable & Finite \\
Quasiperturbatively solvable, QPS & Part & Non-normalizable & Finite \\
\hline \hline
\end{tabular}


and so the square of the angular momentum operator becomes

$$
\mathbf{J}^{2}=-\partial_{\theta}^{2}-\cot \theta \partial_{\theta}-\frac{1}{\sin ^{2} \theta}\left(\partial_{\varphi}^{2}+\partial_{\chi}^{2}\right)+2 \cot \theta \csc \theta \partial_{\varphi} \partial_{\chi} .
$$

Since potential (2) only depends on the polar angle $\theta$, we can separate variables and write the solution, $\psi_{3 \mathrm{D}, t}(\theta, \varphi, \chi)$, to the Schrödinger equation

$$
\mathcal{H}_{t} \psi_{3 \mathrm{D}, t}(\theta, \varphi, \chi)=E_{t} \psi_{3 \mathrm{D}, t}(\theta, \varphi, \chi)
$$

as $\psi_{3 \mathrm{D}, t}(\theta, \varphi, \chi)=\hat{\psi}_{t}(\theta) e^{-i M \varphi} e^{-i K \chi}$, with $M$ and $K$ the (constant) integer projections of $\mathbf{J}$ on the space- and body-fixed axis, respectively. Substitution of the wave function $\psi_{3 \mathrm{D}, t}(\theta, \varphi, \chi)$ into Eq. (9) then leads to the Schrödinger equation

$$
\begin{aligned}
\hat{\mathcal{H}}_{t} \hat{\psi}_{t}(\theta)= & B\left[-\partial_{\theta}^{2}-\cot \theta \partial_{\theta}+\left(M^{2}+K^{2}\right) \csc ^{2} \theta\right. \\
& \left.-2 M K \csc \theta \cot \theta-\rho K^{2}\right] \hat{\psi}_{t}(\theta) \\
& +\left(-\eta \cos \theta-\zeta \cos ^{2} \theta\right) \hat{\psi}_{t}(\theta) \\
= & E_{t} \hat{\psi}_{t}(\theta)
\end{aligned}
$$

for $\hat{\psi}_{t}(\theta)$ which, when gauged ${ }^{2}$ as

$$
\hat{\psi}_{t}(\theta)=\psi_{t}(\theta) / \sqrt{\sin \theta}
$$

allows us to recast our initially three-dimensional eigenproblem as a one-dimensional one

$$
H_{t} \psi_{t}(\theta)=-B \partial_{\theta}^{2} \psi_{t}(\theta)+V_{t}(\theta) \psi_{t}(\theta)=E_{t} \psi_{t}(\theta)
$$

for an effective potential

$$
\begin{aligned}
V_{t}(\theta)= & B\left[\left(M^{2}+K^{2}-\frac{1}{4}\right) \csc ^{2} \theta\right. \\
& \left.-2 M K \cot \theta \csc \theta-\rho K^{2}-\frac{1}{4}\right] \\
& -\eta \cos \theta-\zeta \cos ^{2} \theta
\end{aligned}
$$

We note that, for $K=0$, Eq. (13) yields the spherical pendulum Hamiltonian $[33,59,60,65]$ and, for $(K, M)=(0,1 / 2)$, the planar pendulum Hamiltonian $[32,61]$.

As in our previous work [61], we will also consider the "hyperbolic counterpart" of the above trigonometric symmetric top, obtained by the coordinate transformation $\theta \mapsto i \theta$ and gauging

$$
\psi_{h}(\theta)=\hat{\psi}_{h}(\theta) \sqrt{\sinh \theta}
$$

where the subscript $h$ stands for hyperbolic. This leads to the Schrödinger equation

$$
H_{h} \psi_{h}(\theta)=-B \partial_{\theta}^{2}+V_{h}(\theta) \psi_{h}(\theta)=-E_{t} \psi_{h}(\theta)
$$

with the effective potential

\footnotetext{
${ }^{2}$ For the origin of this gauge transformation see, e.g., Refs. [2,63,64].
}

$$
\begin{aligned}
V_{h}(\theta)= & B\left[\left(M^{2}+K^{2}-\frac{1}{4}\right) \operatorname{csch}^{2} \theta\right. \\
& \left.-2 M K \operatorname{csch} \theta \operatorname{coth} \theta+\rho K^{2}+\frac{1}{4}\right] \\
& +\eta \cosh \theta+\zeta \cosh ^{2} \theta .
\end{aligned}
$$

We note that the transformation $\theta \mapsto i \theta$ is anti-isospectral [61,62], as $E_{t} \mapsto E_{h}:=-E_{t}$.

In what follows, we will refer to the tops described by Schrödinger equations (12) and (15) as the trigonometric and hyperbolic top, respectively.

\section{CONDITIONAL QUASISOLVABILITY}

In this section, we apply the quantum Hamilton-Jacobi theory $[66,67]$ to the trigonometric and hyperbolic top eigenproblems for Hamiltonians (12) and (15), respectively, and construct the closed-form solutions.

Since the derivations for the two types of top are analogous to one another, we show the derivation for the trigonometric top only and subsequently provide a summary of the results for the hyperbolic one.

\section{A. Quantum Hamilton-Jacobi equation}

The Schrödinger equation (12) can be recast as a Ricatti equation

$$
p(\theta)^{2}-i \sqrt{B} p^{\prime}(\theta)=E-V(\theta)
$$

for the quantum momentum function

$$
p(\theta)=-i \sqrt{B} \frac{\psi^{\prime}(\theta)}{\psi(\theta)}
$$

with $\psi^{\prime}(\theta) \equiv \partial_{\theta} \psi(\theta)$, where we dropped the subscripts $t$ or $h$ on $p, E, V$, and $\psi$ for simplicity. Crucially, the wave function $\psi(\theta)$ is assumed to be meromorphic in QHJ theory, i.e., containing at most isolated singularities (poles). Equation (17) is termed the quantum Hamilton-Jacobi equation; in the limit $\hbar \rightarrow 0$, i.e., $B \rightarrow 0$, it turns into the classical Hamilton-Jacobi equation. We note that the quantum momentum function is related to the SUSY QM superpotential $W(\theta)$ via $p(\theta)=$ $i W(\theta)[66]$.

In an appropriate new coordinate $z=z(\theta)$, the quantum Hamilton-Jacobi equation (17) can be transformed into a purely rational form,

$$
\tilde{p}(z)^{2}+\sqrt{B} \tilde{p}^{\prime}(z)+\theta^{\prime}(z)^{2}[E-\tilde{V}(z)]=0,
$$

with a $p(\theta) \mapsto \tilde{p}(z)$ mapping given by Eq. (A1) (for details see Appendix A). This can be considered a normal form [68] of the Riccati equation (17). This rational form will prove key for finding its solutions algebraically via Laurent series expansion of the quantum momentum function in Sec. IIIB. For the explicit choice of $z=(\cos \theta+1) / 2$ as the new variable, ${ }^{3}$

\footnotetext{
${ }^{3}$ We note that a more intuitive choice of the new variable, $z=\cos \theta$, would have no effect on the solution spaces, as it is just a Möbius transform of $z=(\cos \theta+1) / 2$ (see Appendix A). However, it would result in four diagonal elements, whereas our above choice has only three, which is of computational advantage (see Sec. IIID).
} 
the corresponding purely rational potential of the trigonometric top becomes

$$
\tilde{V}_{t}(z)=-\eta(2 z-1)-\zeta(2 z-1)^{2}+B\left(\frac{-4 K^{2} \rho z^{2}+4 K^{2} \rho z-K^{2}+4 K M z-2 K M-M^{2}+\frac{1}{4}}{4(z-1) z}-\frac{1}{4}\right) .
$$

Note that its singularity structure differs from that of the original potential (13): Whereas the original potential $V_{t}(\theta)$ has double poles at $\theta_{m}=m \pi$ on $\mathbb{R}$ with $m$ integer, the transformed potential $\tilde{V}_{t}(z)$ possesses simple poles at the points $z_{1,2} \in\{0,1\}$ in the physical domain $[0,1]$ and its extension, the complex plane $\mathbb{C}$. In addition, it has a double pole at $z_{0}=\infty$. The singularity structure is of consequence for determining the quasisolvability conditions (see Sec. IIIC).

\section{B. Construction of the quantum momentum function}

Given that Hamiltonians (12) and (15) belong to conditionally quasisolvable Hamiltonians in the coordinates $z \propto$ $\cos \theta$ and $z \propto \cosh \theta$, respectively [69], they can be gaugetransformed into $s l(2)$-algebraizable Sturm-Liouville operators $T$ which preserve the finite-dimensional monomial subspaces $^{4}[70]$

$$
\mathcal{P}_{n}=\operatorname{span}\left\{1, z, z^{2}, \ldots, z^{n}\right\} .
$$

As a result, the wave function

$$
\tilde{\psi}(z)=e^{\frac{1}{\sqrt{B}} \int^{z} \tilde{p}(y) d y}
$$

that corresponds to the transformed problem, Eq. (19), and is related to the original wave function via

$$
\psi(\theta)=\left.\tilde{\psi}(z) \sqrt{\theta^{\prime}(z)}\right|_{z=z(\theta)}
$$

[cf. Eq. (A7) of Appendix A], can be factorized into a product of a seed function $\phi_{f}(z)$ and a polynomial $\phi_{m, i}(z)$ :

$$
\tilde{\psi}_{i}(z)=\phi_{f}(z) \phi_{m, i}(z) \quad \text { with } \quad \phi_{m, i}(z) \in \mathcal{P}_{n}
$$

pertaining to eigenenergy $E_{i}$. For $n=0, \tilde{\psi}_{0}(z) \propto \phi_{f}(z)$, from which we see that the seed function $\phi_{f}(z)$ determines the algebraic sector of the solutions, i.e., the class of wave functions which only differ from one another by the polynomial $\phi_{m, i}(z)$ in Eq. (24). Hence the seed function can serve as a gauge factor which allows the transformation of Hamiltonians (12) and (15) to the Sturm-Liouville self-adjoint operators [55,69]

$$
T_{t, h}(z)=\left.\frac{1}{\phi_{f}(z)} H_{t, h}(\theta) \phi_{f}(z)\right|_{\theta=\theta(z)}
$$

with the subscripts $t$ and $h$ referring to the trigonometric and hyperbolic top, respectively. ${ }^{5}$

By combining Eqs. (22) and (24), we can decompose the quantum momentum function as

$$
\tilde{p}(z)=\tilde{p}_{f}(z)+\tilde{p}_{m}(z)
$$

\footnotetext{
${ }^{4}$ The monomial subspaces form the flag $\mathcal{P}_{0} \subset \mathcal{P}_{1} \subset \ldots \subset \ldots \subset$ $\mathcal{P}_{n}$. If the Hamiltonian preserved a complete flag $\mathcal{P}_{0} \subset \mathcal{P}_{1} \subset \ldots \subset$ $\ldots \subset \mathcal{P}_{n} \subset \ldots$, it would be said to be exactly solvable.

${ }^{5}$ That the seed function is a meromorphic and algebraic function of $z$ forces $T_{t, h}$ of Eq. (25) to preserve the span (21) and to be self-adjoint.
}

with

$$
\tilde{p}_{f}(z)=\sqrt{B} \partial_{z} \phi_{f}(z) / \phi_{f}(z)
$$

and

$$
\tilde{p}_{m}(z)=\sqrt{B} \partial_{z} \phi_{m, i}(z) / \phi_{m, i}(z)
$$

Clearly, if $\tilde{p}_{f}(z)$ is known, the seed function can be determined via $\phi_{f}(z)=e^{\frac{1}{\sqrt{B}} \int^{z} \tilde{p}_{f}(y) d y}$.

Our notation above, starting with Eq. (24), anticipates the fact that the quantum momentum function $\tilde{p}(z)$ can have two types of poles: fixed poles (subscript $f$ ) that are due to the poles of $\tilde{V}(z)$ and $\theta^{\prime}(z)^{2}$ of Eq. (19), and moving poles (subscript $m$ ) that are due to the nodes of the wave function. Thus the notation accounts for the fact that $\tilde{p}_{f}(z)$, the part of the quantum momentum function that pertains to the seed function, has fixed poles, and that $\tilde{p}_{m}(z)$, the part of the quantum momentum function that pertains to polynomials in $z$, has moving poles. As noted above, the fixed poles occur at $z_{j} \in\{\infty, 0,1\}$.

We will now determine the closed-form expressions for the quantum momentum function term $\tilde{p}_{f}(z)$ that pertains to the fixed poles.

By setting $\phi_{m, i}=$ const. in Eq. (27), which corresponds to the lowest-order solution $\tilde{\psi}_{0}$ with $n=0$, Eq. (27) yields $\tilde{p}_{m, i}=0$ and Eq. (26) yields $\tilde{p}=\tilde{p}_{f}$. As a result, the quantum Hamilton-Jacobi equation (19) reduces to

$$
\tilde{p}_{f}(z)^{2}+\sqrt{B} \tilde{p}_{f}^{\prime}(z)+\theta^{\prime}(z)^{2}[E-\tilde{V}(\theta(z))]=0 .
$$

Provided $\tilde{V}(z)$ is a rational function of $z$ [see Eq. (20)], $\tilde{p}_{f}(z)$ must be rational as well and can therefore be decomposed into partial fractions $\tilde{p}_{f, j}$, each of which is evaluated at one of the poles $z_{j} \in\{\infty, 0,1\}$ :

$$
\tilde{p}_{f}(z)=\sum_{j=0}^{\#\left\{z_{j}\right\}-1} \tilde{p}_{f, j}(z)
$$

with $\#\left\{z_{j}\right\}$ the number of fixed poles $z_{j}$.

For $j=1,2$, i.e., at $z_{1,2} \in\{0,1\}$, each of the components $\tilde{p}_{f, j}$ can be expanded in terms of a Laurent series

$$
\tilde{p}_{f, j}(z)=\sum_{k=-d}^{\infty} c_{j k}\left(z-z_{j}\right)^{k}
$$

with $d \in \mathbb{N}_{\geqslant 0}$ a sufficiently large finite boundary. 
TABLE III. Quantum momentum function terms $\tilde{p}_{f}$ and $\hat{p}_{f, 0}$ for each algebraic sector.

\begin{tabular}{lcc}
\hline \hline Sector & \multicolumn{1}{c}{$\tilde{p}_{f}(z)$} & $\hat{p}_{f, 0}(w)$ \\
\hline $1_{ \pm}$ & $\sqrt{B}\left(\frac{-K+M+1}{2(z-1)}+\frac{K+M+1}{2 z}\right) \pm 2 \sqrt{\zeta}$ & $\mp \frac{\eta w}{2 \sqrt{\zeta}} \pm 2 \sqrt{\zeta}$ \\
$2_{ \pm}$ & $\sqrt{B}\left(\frac{K-M+1}{2(z-1)}+\frac{K+M+1}{2 z}\right) \pm 2 \sqrt{\zeta}$ & $\mp \frac{\eta w}{2 \sqrt{\zeta}} \pm 2 \sqrt{\zeta}$ \\
$3_{ \pm}$ & $\sqrt{B}\left(\frac{-K+M+1}{2(z-1)}+\frac{-K-M+1}{2 z}\right) \pm 2 \sqrt{\zeta}$ & $\mp \frac{\eta w}{2 \sqrt{\zeta}} \pm 2 \sqrt{\zeta}$ \\
$4_{ \pm}$ & $\sqrt{B}\left(\frac{K-M+1}{2(z-1)}+\frac{-K-M+1}{2 z}\right) \pm 2 \sqrt{\zeta}$ & $\mp \frac{\eta w}{2 \sqrt{\zeta}} \pm 2 \sqrt{\zeta}$ \\
\hline \hline
\end{tabular}

In order to find the coefficients $c_{j k}$ of Eq. (30), we plug each $\tilde{p}_{f, j}$ into Eq. (28) separately:

$$
\begin{aligned}
\tilde{p}_{f, j}(z)^{2}+\sqrt{B} \tilde{p}_{f, j}{ }^{\prime}(z)+\frac{E}{z-z^{2}} \\
+\frac{\eta(1-2 z)}{(z-1) z}-\frac{\zeta(1-2 z)^{2}}{(z-1) z} \\
\quad+B\left(\frac{K^{2}[-4 r(z-1) z-1]+2 K M(2 z-1)-M^{2}+1}{4(z-1)^{2} z^{2}}\right) \\
=0 .
\end{aligned}
$$

At $z_{0}=\infty$, however, the evaluation of $\tilde{p}_{f, 0}$ from Eq. (29) requires another extension of the domain: As illustrated in Fig. 1, we have to add a point at infinity by introducing a copy of the complex plane $\mathbb{C}$ with coordinates $w=1 / z$, which we denote as $\mathcal{D}_{c}(w)$. This compactifies $\mathbb{C}$, so that $\mathcal{D}_{c}(1 / w) \cup \mathcal{D}_{c}(w)$ becomes a cover of the Riemann sphere

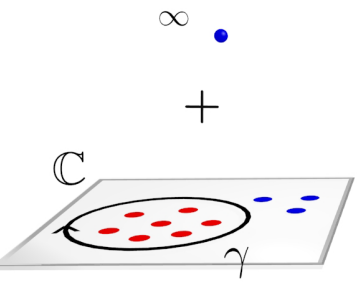

(a) (b)

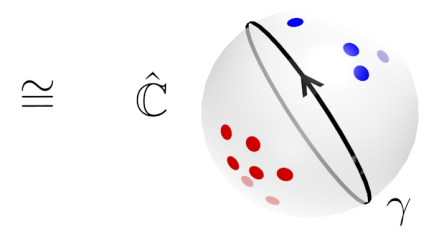

FIG. 1. (a) Extension of the domain $\mathcal{D}_{p}(z)$ of the potential $\tilde{V}_{t}(z)$, Eq. (20), to the complex plane $\mathbb{C}$; fixed poles are depicted by the blue region and the $n$ moving poles are depicted by the red region. (b) Compactification of the complex plane to the Riemann sphere $\hat{\mathbb{C}}$ by adding a point at infinity shown in panel (a). A contour integral of $\tilde{p}_{m}(z)$ along the path $\gamma$ enclosing all moving poles (red) is equal to the contour integral of $\tilde{p}_{f}(z)$ along $\gamma$ in the opposite direction enclosing all fixed poles (blue).

$\hat{\mathbb{C}} \cong \mathbb{C} \cup\{\infty\}$ [71]. The inversion $w=1 / z$ allows for a reciprocal mapping between the elements of $\mathcal{D}_{c}(z)$ and $\mathcal{D}_{c}(w)$. By introducing $\hat{p}_{f}(w(z))=\tilde{p}_{f}(z)$, we obtain the Laurent series expansion of the component $\tilde{p}_{f, 0}(z)$ in Eq. (29):

$$
\tilde{p}_{f, 0}(z)=\hat{p}_{f, 0}(w(z))=\sum_{k=-d}^{\infty} c_{0 k} w(z)^{k} .
$$

Similarly to the case of the coefficients $c_{1 k}$ and $c_{2 k}$ above, the coefficients $c_{0 k}$ can be found by inserting $\hat{p}_{f, 0}(w)$ into the Riccati equation $(28)$ on $\mathcal{D}_{c}(w):^{6}$

$$
\begin{gathered}
\hat{p}_{f, 0}(w)^{2}-\sqrt{B} w^{2} \hat{p}_{f, 0}{ }^{\prime}(w)-\frac{\eta w(w-2)}{w-1}+\frac{\zeta(w-2)^{2}}{w-1}+\frac{E w^{2}}{w-1} \\
-\frac{B w^{2}\left(K^{2}\left[w^{2}-4 \rho(w-1)\right]+2 K M(w-2) w+\left(M^{2}-1\right) w^{2}\right)}{4(w-1)^{2}}=0
\end{gathered}
$$

with $\lim _{z \rightarrow \infty} \tilde{p}_{f, 0}(z)=\lim _{w \rightarrow 0} \hat{p}_{f, 0}(w)=$ const. From the domain compactification we find that $\tilde{p}_{f, 0}(z)$ on $\mathcal{D}_{c}(z)$ is bounded by $\hat{p}_{f, 0}(w)$ at $w=0$ on $\mathcal{D}_{c}(w)$. From Liouville's theorem of complex analysis [72], it then follows that $\tilde{p}_{f, 0}(z)=$ const. As a result, Eq. (29) boils down to

$$
\tilde{p}_{f}(z)=\hat{p}_{f, 0}(0)+\sum_{j=1}^{\#\left\{z_{j}\right\}-1} \tilde{p}_{f, j}(z)
$$

Finally, inserting the series expansion (30) of $\tilde{p}_{f, 1}$ and $\tilde{p}_{f, 2}$ at the poles $z_{1}=0$ and $z_{2}=1$ into Eq. (31) and likewise the series expansion (32) of $\hat{p}_{f, 0}(w(z))$ at the pole at $z_{0}=\infty$ into Eq. (33) completely determines $\tilde{p}_{f}$ in (34) as the exact and closed-form solution of the quantum Hamilton-Jacobi equation (28).

Due to the squared $\tilde{p}_{f, j}$ term in Eqs. (31) and (33), a branching and hence at least two possible solutions $\tilde{p}_{f, j}$ can be expected for each $z_{j}$ resulting in $2^{\#\left\{z_{j}\right\}}$ combinations and as many solutions $\tilde{p}_{f}$. And indeed, for the coordinate choice

\footnotetext{
${ }^{6} \mathrm{Here}$, we bypass the normal form of the Riccati equation, since $z \mapsto 1 / z$ is a Möbius transformation and hence does not yield any nonrational terms.
}

$z=(\cos \theta+1) / 2$ we find exactly $2^{3}$ algebraic sectors (see Table III).

\section{Conditions of quasisolvability}

We will now derive the conditions for quasisolvability (see Ref. [58]) and with their help the polynomial multipliers $\phi_{m, i}(z)$ of the seed functions in the factorized algebraic wave functions $\tilde{\psi}_{i}(z)$ via Eq. (24).

The complexification of $z$, introduced in Sec. IIIB, allows us to define the contour integral of the part $\tilde{p}_{m}(z)=$ $\sqrt{B} \phi_{m, i}^{\prime}(z) / \phi_{m, i}(z)$ of the quantum momentum function $\tilde{p}(z)$ of Eq. (26) along the curve $\gamma$ enclosing the $n$ moving poles (see the red dots in Fig. 1). By Cauchy's argument principle [72], this is equivalent to the sum of the corresponding residues,

$$
\frac{1}{2 \pi i} \oint_{\gamma} \tilde{p}_{m} d z=\sqrt{B} \sum_{j=0}^{n} \operatorname{Res}\left(\frac{\phi_{m, i}^{\prime}(z)}{\phi_{i}(z)}, z_{j}\right)=\sqrt{B} n,
$$

where $\operatorname{Res}\left(f(z), z_{j}\right)$ stands for the residue of a function $f(z)$ at its pole $z_{j}$. Let $z$ be an element of the compactified complex plane $\hat{\mathbb{C}}$. This allows us to redefine the contour integral (35) on the Riemann sphere (see Fig. 1) and to identify it with the contour integral of $\tilde{p}_{f}(z)$ and $\hat{p}_{f, 0}(1 / z)$ along $\gamma$ in the opposite 
TABLE IV. The residues of the quantum momentum function and the quasisolvability (QS) conditions for each algebraic sector for the trigonometric and hyperbolic top with coordinates $z=(\cos \theta+1) / 2$ and $(\cosh \theta+1) / 2$, respectively.

\begin{tabular}{lcccc}
\hline \hline Sector & $\operatorname{Res}\left(\hat{p}_{f}, \infty\right)$ & $\operatorname{Res}\left(\tilde{p}_{f}, 0\right)$ & $\operatorname{Res}\left(\tilde{p}_{f}, 1\right)$ & QS conditions \\
\hline $1_{ \pm}$ & $\mp \frac{\eta}{2 \sqrt{\zeta}}$ & $\frac{1}{2} \sqrt{B}(K+M+1)$ & $\frac{1}{2} \sqrt{B}(-K+M+1)$ & $\eta= \pm 2 \sqrt{B}(M+n+1) \sqrt{\zeta}$ \\
$2_{ \pm}$ & $\mp \frac{\eta}{2 \sqrt{\zeta}}$ & $\frac{1}{2} \sqrt{B}(K+M+1)$ & $\frac{1}{2} \sqrt{B}(K-M+1)$ & $\eta= \pm 2 \sqrt{B}(K+n+1) \sqrt{\zeta}$ \\
$3_{ \pm}$ & $\mp \frac{\eta}{2 \sqrt{\zeta}}$ & $\frac{1}{2} \sqrt{B}(-K-M+1)$ & $\frac{1}{2} \sqrt{B}(-K+M+1)$ & $\eta= \pm 2 \sqrt{B}(-K+n+1) \sqrt{\zeta}$ \\
$4_{ \pm}$ & $\mp \frac{\eta}{2 \sqrt{\zeta}}$ & $\frac{1}{2} \sqrt{B}(-K-M+1)$ & $\frac{1}{2} \sqrt{B}(K-M+1)$ & $\eta= \pm 2 \sqrt{B}(-M+n+1) \sqrt{\zeta}$ \\
\hline \hline
\end{tabular}

direction (see Theorem 2.2 of Ref. [73]) enclosing all fixed poles, $z_{j} \in\{\infty, 0,1\}$, and represented by the blue region in Fig. 1:

$$
\begin{aligned}
&- \frac{1}{2 \pi i} \oint_{\gamma}\left[\tilde{p}_{f}(z)+\hat{p}_{f, 0}(1 / z)\right] d z \\
&=-\operatorname{Res}\left(\hat{p}_{f, 0}(1 / z), 0\right)-\sum_{j=1}^{\#\left\{z_{j}\right\}-1} \operatorname{Res}\left(\tilde{p}_{f, j}, z_{j}\right)=\sqrt{B} n
\end{aligned}
$$

with $\operatorname{Res}\left(\hat{p}_{f, 0}(1 / z), 0\right)=-\operatorname{Res}\left(\frac{1}{w^{2}} \hat{p}_{f, 0}(w), 0\right)$.

Equation (36), which can be regarded as a "quantization condition," leads ultimately to the conditions of quasisolvability. All we need to do is to evaluate the residues $\operatorname{Res}\left(\tilde{p}_{f}, 0\right)$, $\operatorname{Res}\left(\tilde{p}_{f}, 1\right)$, and $\operatorname{Res}\left(\hat{p}_{f, 0}, 0\right)$ from the quantum momentum functions listed in Table III. The conditions of quasisolvability then follow via Eq. (36) and are listed in Table IV. One can see that these fix the admissible ratios of the interaction parameters $\eta$ and $\zeta$, conveniently expressed in terms of the topological index, $\kappa=\frac{\eta}{\sqrt{B \zeta}}$, introduced in our earlier work [32]. As discussed in Sec. IV and shown in Figs. 2-5, the topological

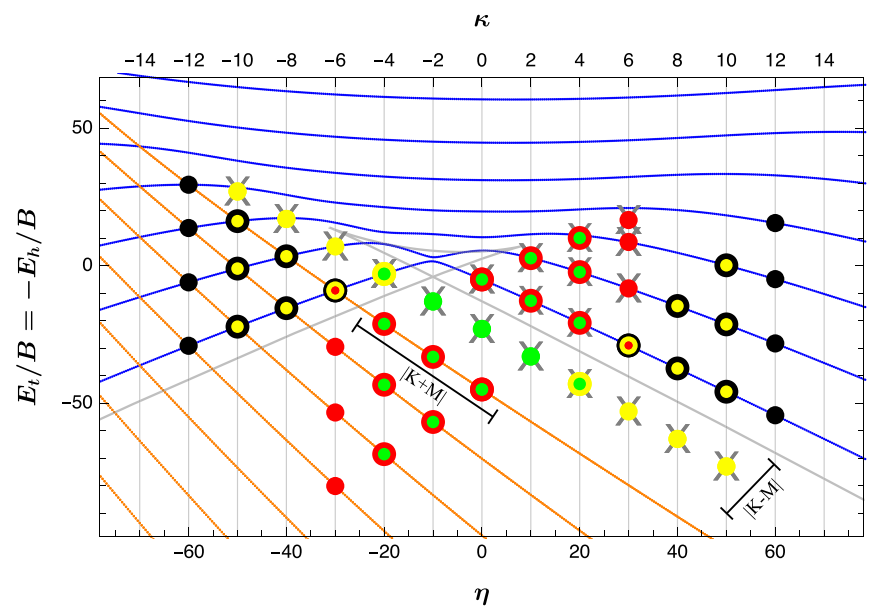

FIG. 2. Numerical spectra for $M=2, K=1$ and $\zeta=25, \rho=0$ : trigonometric top (blue), hyperbolic top (orange). Black, sector $1_{+}$ (here $\kappa \geqslant 6)$, sector $1_{-}(\kappa \leqslant-6)$; yellow, sector $2_{+}(\kappa \geqslant 4)$, sector $2_{-}(\kappa \leqslant-4)$; red, sector $3_{+}(\kappa \geqslant 0)$, sector $3_{-}(\kappa \leqslant 0)$; green, sector $4_{+}(\kappa \geqslant-2)$, sector $4_{-}(\kappa \leqslant 2)$. Gray crosses mark nonnormalizable solutions. Gray curves show local minima and maxima of the symmetric top potential, Eq. (13). For computational details, see Appendices B and C. index allows us to label the loci of the level crossings as well as the closed-form solutions obtained.

We note that the residues and quasisolvability conditions for the hyperbolic top with $z=(\cosh \theta+1) / 2$ coincide with those for the trigonometric top with $z=(\cos \theta+1) / 2$.

\section{Matrix elements}

By substituting the decomposition (26) into Eq. (19) and multiplying by $1 / \theta^{\prime}(z)^{2}$, one obtains the following eigenproblem:

$$
\begin{aligned}
& \left(T_{t}+E_{i}\right) \phi_{m, i}(z)=B \frac{1}{\theta^{\prime}(z)^{2}}\left[\phi_{m, i}^{\prime \prime}(z)+2 \sqrt{B} \tilde{p}_{f}(z) \phi_{m, i}^{\prime}(z)\right] \\
& +\left[\frac{1}{\theta^{\prime}(z)^{2}}\left[\tilde{p}_{f}(z)^{2}+\sqrt{B} \tilde{p}_{f}^{\prime}(z)\right]+E_{i}-\tilde{V}_{t}(\theta(z))\right] \phi_{m, i}(z) \\
& =0
\end{aligned}
$$

with the operator $T_{t}$ defined by Eq. (25) and fulfilling $T_{t}=$ $-T_{h}$. Substituting for the quantum momentum functions $\tilde{p}_{f}$ from Table IV then yields eight self-adjoint operators $T_{t}$, each corresponding to a particular algebraic sector. The same applies to the hyperbolic analog $T_{h}$.

The matrix elements

$$
\left(T_{t}\right)_{k \ell}=\left\langle z^{k}\left|T_{t}\right| z^{\ell}\right\rangle
$$

of the Sturm-Liouville operator $T_{t}$ in the basis set of the monomials in $z$, Eq. (21), can be constructed either directly or via the residues

$$
\left\langle z^{k}\left|T_{t}\right| z^{\ell}\right\rangle=\operatorname{Res}\left(\frac{1}{z} \times \frac{1}{z^{k}} T_{t}\left(z^{\ell}\right), 0\right)
$$

which provide the constant part of $\left(1 / z^{k}\right) T_{t}\left(z^{\ell}\right)$.

Hence the eight Sturm-Liouville operators $T_{t}$ are each represented by a tridiagonal matrix the elements of which are listed in Tables V and VI. Note that the integer $n$ in Table IV determines the cutoff dimension at which a given matrix can be decomposed into an (upper) finite $n \times n$ block and a (lower) infinite-dimensional block [61]. Hence, the closed-form solutions can be constructed for $k, \ell \leqslant n$. Solutions beyond the $n$-dimensional block can only be determined numerically and require multiprecision computations. 


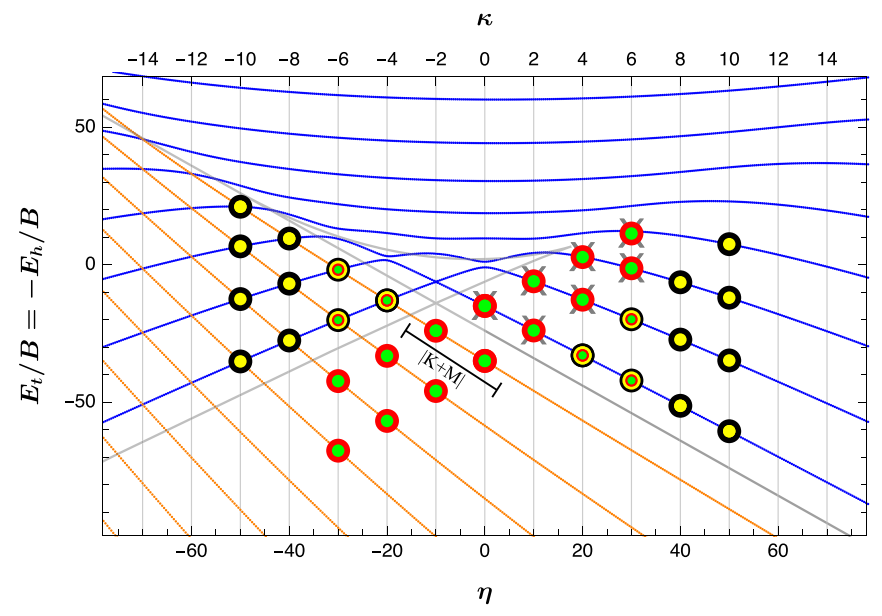

FIG. 3. Numerical spectra for $M=1, K=1$ and $\zeta=25, \rho=$ 0 : trigonometric top (blue), hyperbolic top (orange). Black, sector $1_{+}$(here $\left.\kappa \geqslant 4\right)$, sector $1_{-}(\kappa \leqslant-4)$; yellow, sector $2_{+}(\kappa \geqslant 4)$, sector $2_{-}(\kappa \leqslant-4)$; red, sector $3_{+}(\kappa \geqslant 0)$, sector $3_{-}(\kappa \leqslant 0)$; green, sector $4_{+}(\kappa \geqslant 0)$, sector $4_{-}(\kappa \leqslant 0)$. Gray crosses mark non-normalizable solutions. Gray curves show local minima and maxima of the symmetric top potential, Eq. (13). For computational details, see Appendices B and C.

\section{E. Closed-form wave functions}

The lowest-order closed-form wave functions, i.e., the seed functions for $n=0$, are found by making use of Eqs. (A8) and (24) and the algebraic quantum momentum functions listed in Table III:

$\left.\psi_{t, 0}(\theta) \propto \phi_{m, 0}(z) \phi_{f}(z) \sqrt{\partial_{z} \theta(z)}\right|_{z=z(\theta)}$

with $\phi_{f}(z)=e^{\frac{1}{\sqrt{B}} \int^{z} \tilde{p}_{f}(y) d y} \quad$ and $\phi_{m, 0}=$ const.

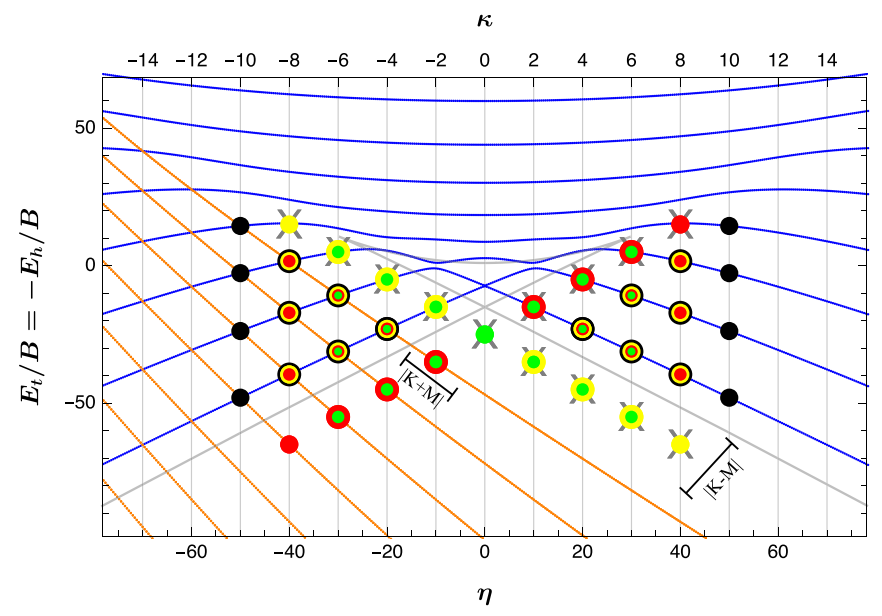

FIG. 4. Numerical spectra for $M=1, K=0$ and $\zeta=25, \rho=$ 0: trigonometric top (blue), hyperbolic top (orange). Black, sector $1_{+}$(here $\left.\kappa \geqslant 4\right)$, sector $1_{-}(\kappa \leqslant-4)$; yellow, sector $2_{+}(\kappa \geqslant 2)$, sector $2_{-}(\kappa \leqslant-2)$; red, sector $3_{+}(\kappa \geqslant 2)$, sector $3_{-}(\kappa \leqslant-2)$; green, sector $4_{+}(\kappa \geqslant 0)$, sector $4_{-}(\kappa \leqslant 0)$. Gray crosses mark nonnormalizable solutions. Gray curves show local minima and maxima of the spherical pendulum potential, Eq. (13). For computational details, see Appendices B and C.

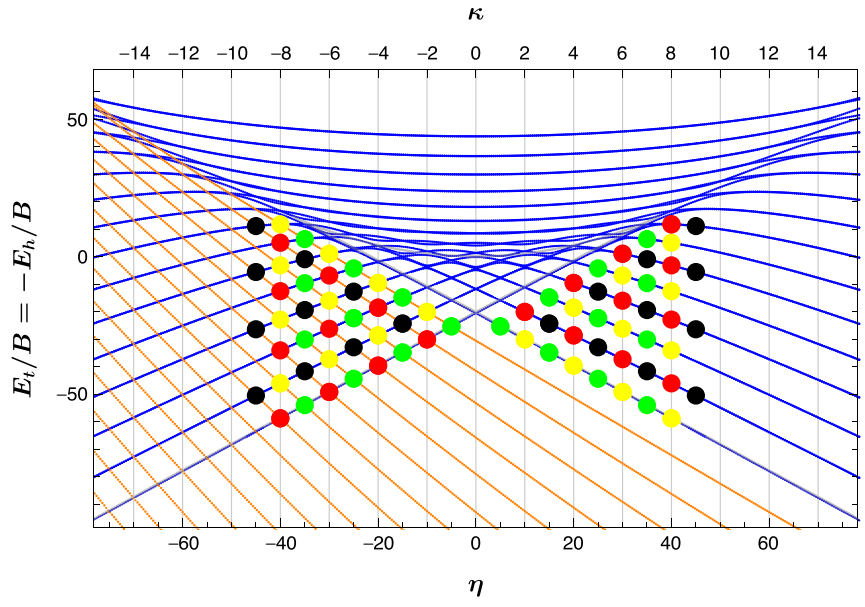

FIG. 5. Numerical spectra for $M=1 / 2, K=0$ and $\zeta=25, \rho=$ 0 : trigonometric top and planar pendulum (blue), hyperbolic top and Razavy system (orange). Black, sector $1_{+}$(here $\kappa \geqslant 3$ ), sector $1_{-}$ $(\kappa \leqslant-3)$; yellow, sector $2_{+}(\kappa \geqslant 2)$, sector $2_{-}(\kappa \leqslant-2)$; red, sector $3_{+}(\kappa \geqslant 2)$, sector $3_{-}(\kappa \leqslant-2)$; green, sector $4_{+}(\kappa \geqslant 1)$, sector $4_{-}$ $(\kappa \leqslant-1)$. Gray curves show the local minima and maxima of the trigonometric top potential, Eq. (13). For computational details, see Appendices B and C.

Then the explicit expressions for the wave functions $\hat{\psi}_{t, 0}(\theta)=$ $\psi_{t, 0}(\theta) / \sqrt{\sin \theta}$, Eq. (11), read

$$
\hat{\psi}_{t, 0}(\theta)=e^{ \pm \sqrt{\zeta / B} \cos (\theta)} \sin ^{ \pm_{1} K+\mp_{1} M}\left(\frac{\theta}{2}\right) \cos ^{ \pm_{2} K+ \pm_{2} M}\left(\frac{\theta}{2}\right),
$$

where the signs are to be set with respect to the algebraic sectors. Note that the dimension of an algebraic solution subspace is fixed to $n+1$, so that $i$ labels the $n+1$ available algebraic solutions per algebraic sector.

A list of all closed-form wave functions

$$
\hat{\psi}_{t, i}(\theta)=\left.\hat{\psi}_{t, 0}(\theta) \phi_{m, i}(z)\right|_{z=z(\theta)}
$$

which solve Schrödinger's Eq. (10) for the trigonometric symmetric top determined via Eqs. (24) and (41) [the coefficients in $\phi_{m, i}(z)$ are fixed by the eigenvectors of the matrix elements in Tables V and VI] is given in Table VII for $n=0,1$. Note that wave functions pertaining to sectors $1_{-}$to $4_{-}$differ from those pertaining to sectors $1_{+}$to $4_{+}$by the change of branch, $\sqrt{\zeta} \mapsto-\sqrt{\zeta}$. According to the Abel-Ruffini theorem [74], the existence of algebraic solutions is not ensured for the characteristic polynomials of degree higher than 4 , although for $n>4$ certain classes of characteristic polynomials may

TABLE V. Matrix elements on the super- and subdiagonal for each algebraic sector.

\begin{tabular}{lcc}
\hline \hline Sector & $\left\langle z^{\ell-1}\left|T_{t}\right| z^{\ell}\right\rangle$ & $\left\langle z^{\ell}\left|T_{t}\right| z^{\ell-1}\right\rangle$ \\
\hline $1_{ \pm}$ & $B \ell(K+M+\ell)$ & $2 \eta \mp 4 \sqrt{B} \sqrt{\zeta}(M+\ell)$ \\
$2_{ \pm}$ & $B \ell(K+M+\ell)$ & $2 \eta \mp 4 \sqrt{B} \sqrt{\zeta}(K+\ell)$ \\
$3_{ \pm}$ & $B \ell(-K-M+\ell)$ & $2 \eta \mp 4 \sqrt{B} \sqrt{\zeta}(-K+\ell)$ \\
$4_{ \pm}$ & $B \ell(-K-M+\ell)$ & $2 \eta \mp 4 \sqrt{B} \sqrt{\zeta}(-M+\ell)$ \\
\hline \hline
\end{tabular}


TABLE VI. Matrix elements on the main diagonal for each algebraic sector.

\begin{tabular}{lc}
\hline \hline Sector & $\left\langle z^{\ell}\left|T_{t}\right| z^{\ell}\right\rangle$ \\
\hline $1_{ \pm}$ & $-\eta-B\left(M^{2}+2 M \ell+M+\ell(\ell+1)-\rho K^{2}\right) \pm 2 \sqrt{B} \sqrt{\zeta}(K+M+2 \ell+1)+\zeta$ \\
$2_{ \pm}$ & $-\eta-B\left(K^{2}+2 K \ell+K+\ell(\ell+1)-\rho K^{2}\right) \pm 2 \sqrt{B} \sqrt{\zeta}(K+M+2 \ell+1)+\zeta$ \\
$3_{ \pm}$ & $-\eta-B\left(K^{2}-2 K \ell-K+\ell(\ell+1)-\rho K^{2}+\right) \pm 2 \sqrt{B} \sqrt{\zeta}(-K-M+2 \ell+1)+\zeta$ \\
$4_{ \pm}$ & $-\eta-B\left(M^{2}-2 M \ell-M+\ell(\ell+1)-\rho K^{2}\right) \pm 2 \sqrt{B} \sqrt{\zeta}(-K-M+2 \ell+1)+\zeta$ \\
\hline \hline
\end{tabular}

yield further exact, but not necessarily closed-form, solutions $[75,76]$.

The construction of the solutions $\hat{\psi}_{h, i}(\theta)$ of Eq. (15) for the hyperbolic top via the quantum Hamilton-Jacobi theory amounts to applying the general mappings ${ }^{7}$

$$
\cos (\cdot) \mapsto \cosh (\cdot) \quad \text { and } \quad \sin (\cdot) \mapsto \sinh (\cdot),
$$

to the trigonometric solutions $\hat{\psi}_{t, i}(\theta)$ in Eq. (42) and Table VII. The existence of the solutions $\hat{\psi}_{h, i}(\theta)$ is guaranteed by the choice of the coordinate $z=(\cosh \theta) / 2$ (or its Möbius transformations).

The sufficient conditions for the normalizability of the closed-form solutions $\hat{\psi}_{t, i}(\theta)$ of the trigonometric and hyperbolic top are summarized in Table VIII.

Furthermore, we find that the seed functions $\hat{\psi}_{t, 0}(\theta)$ or $\hat{\psi}_{h, 0}(\theta)$ which satisfy one of the normalizability conditions cause all higher-order solutions in the same sector, i.e., $\hat{\psi}_{t, i}(\theta)$ or $\hat{\psi}_{h, i}(\theta)$ for $n>0$, to be normalizable as well. However, the converse holds only for the hyperbolic top. But if for the trigonometric top a higher-order solution $\hat{\psi}_{t, i}(\theta)$ for $n>0$ turns out to be normalizable, then either the seed function of the same sector satisfies one of the above normalizability conditions or it is, up to the phase $e^{i \pi}$, identical with a solution of another sector the seed function of which is normalizable. This situation is illustrated in Figs. $2-4$ for the cases $(K, M)=$ $(1,2),(K, M)=(1,1)$, and $(K, M)=(0,1)$ of Sec. IV.

\section{F. Limit-point and limit-circle classification}

\section{Trigonometric top}

In this section, we discuss the underlying physical and mathematical structure that leads to the existence and/or absence of (non-)normalizable closed-form solutions in Table VII. In particular, we show that if an effective centrifugal potential, that we identify in Eq. (45), remains bounded, then any solution to Eq. (12) is square integrable. Conversely, there exist non-normalizable solutions to Eq. (12) only if this effective centrifugal potential is unbounded at the end points $\theta=0$ or $\pi$. As shown in Table VIII, only some solutions found by the quantum Hamilton-Jacobi method are normalizable and qualify therefore as physical states for the pendulum. In particular, we see that transitions $M=K$ or $-K$ are critical for the normalizability of the solutions presented in Table VII. To understand these properties better, we start with some simple

\footnotetext{
${ }^{7}$ These are not to be confused with the anti-isospectral transformation, which yields a phase shift such that $\hat{\psi}_{t, i}(i \theta)=$ $e^{i \frac{\pi}{2}( \pm K \mp M)} \hat{\psi}_{h, i}(\theta)$.
}

observations for Eq. (10):

$$
\begin{aligned}
\hat{\mathcal{H}}_{t} \hat{\psi}_{t}(\theta)= & B\left[-\partial_{\theta}^{2}-\cot \theta \partial_{\theta}+\left(M^{2}+K^{2}\right) \csc ^{2} \theta\right. \\
& \left.-2 M K \csc \theta \cot \theta-\rho K^{2}\right] \hat{\psi}_{t}(\theta) \\
& +\left(-\eta \cos \theta-\zeta \cos ^{2} \theta\right) \hat{\psi}_{t}(\theta) \\
= & E_{t} \hat{\psi}_{t}(\theta) .
\end{aligned}
$$

Note that normalizable solutions to Eq. (44) coincide with normalizable solutions to Eq. (12) when gauged according to Eq. (11). The term

$$
V_{\text {cent }, \mathrm{t}}(\theta)=\left(M^{2}+K^{2}\right) \csc ^{2} \theta-2 M K \csc \theta \cot \theta
$$

is an effective centrifugal potential and is the decisive quantity for the presence or absence of normalizable solutions to Eq. (44) as we explain now. In particular, the discussion of normalizability is independent of $\eta$ and $\zeta$ for the trigonometric problem because the trigonometric potential, as defined in Eq. (2), is a bounded function. We observe that exactly when $M \neq K$ the centrifugal potential $V_{\text {cent,t }}$ is confining as $\theta \downarrow 0$, i.e.,

$$
\lim _{\theta \downarrow 0} V_{\text {cent,t }}(\theta)=\infty \text {. }
$$

A similar result is true if $M \neq-K$ at $\theta=\pi$ : Unless $M=-K$, the centrifugal potential $V_{\text {cent,t }}$ is confining as $\theta \uparrow \pi$, i.e.,

$$
\lim _{\theta \uparrow \pi} V_{\text {cent,t }}(\theta)=\infty \text {. }
$$

Like any second-order differential equation of PicardLindelöf type, Eq. (44) possesses for any $E_{t} \in \mathbb{C}$ two linearly independent solutions that we denote by $\hat{\psi}_{t}$ and $\hat{\varphi}_{t}$. Given one solution to Eq. (44), which we assume without loss of generality to be $\hat{\psi}_{t}$, a linearly independent partner solution $\hat{\varphi}_{t}$ can be computed from [77]

$$
\hat{\varphi}_{t}(\theta)=\hat{\psi}_{t}(\theta) \int_{\frac{\pi}{2}}^{\theta} \frac{d t}{\sin (t) \hat{\psi}_{t}(t)^{2}} .
$$

In other words, we have that $\left(\hat{\mathcal{H}}_{t} \hat{\psi}_{t}\right)(\theta)=E_{t} \hat{\psi}_{t}(\theta)$ and $\left(\hat{\mathcal{H}}_{t} \hat{\varphi}_{t}\right)(\theta)=E_{t} \hat{\varphi}_{t}(\theta)$ with $\hat{\psi}_{t}$ and $\hat{\varphi}_{t}$ linearly independent. In particular, Eq. (48) allows us to compute a linearly independent second solution to the same energy for any closed-form solution shown in Table VII. Yet, we will see that these solutions do not correspond to physical states of the system.

If $M \neq K$, i.e., confining centrifugal potential in the sense of Eq. (46) at $\theta=0$, then one of the solutions $1_{+}, n=0, i=0$ and $2_{+}, n=0, i=0$ from Table VII,

$$
\hat{\psi}_{t, 0}(\theta)=e^{\sqrt{\zeta / B} \cos (\theta)} \sin ^{ \pm(M-K)}\left(\frac{\theta}{2}\right) \cos ^{M+K}\left(\frac{\theta}{2}\right),
$$

is not square integrable in any neighborhood of $\theta=0$. Similarly, if $M \neq-K$, i.e., confining centrifugal potential at $\theta=\pi$ 


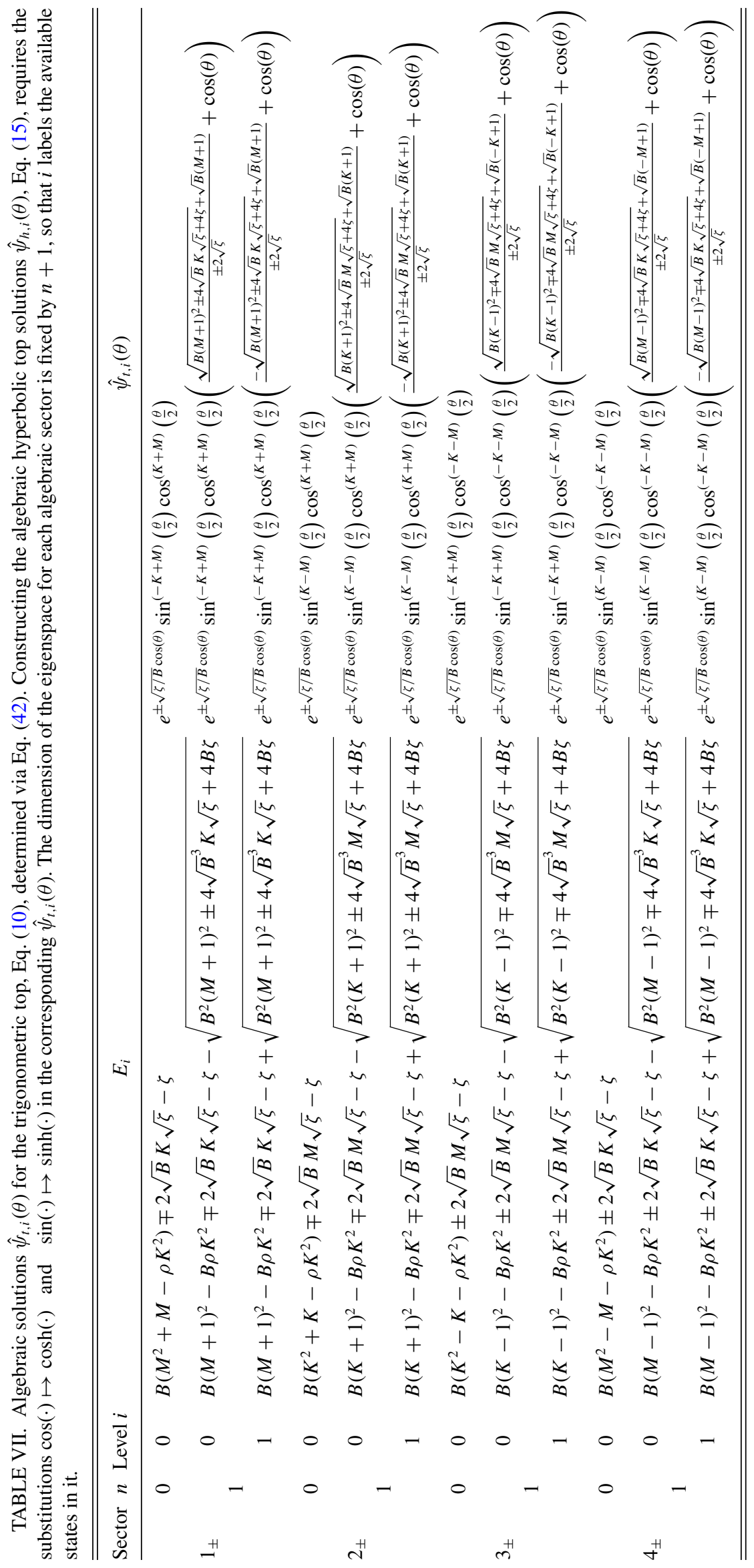


TABLE VIII. Sufficient conditions for the normalizability of the wave functions $\hat{\psi}_{t, i}(\theta)$ and $\hat{\psi}_{h, i}(\theta)$ of the trigonometric and hyperbolic top, respectively.

\begin{tabular}{lclll}
\hline \hline & \multicolumn{2}{c}{ Trigonometric top } & & \multicolumn{2}{c}{ Hyperbolic top } \\
\cline { 5 - 6 } Sector & Condition & & Sector & Condition \\
\hline $1_{ \pm}$ & $K \leqslant M \wedge K \geqslant-M$ & & $1_{-}$ & $K \leqslant M$ \\
$2_{ \pm}$ & $K \geqslant M \wedge K \geqslant-M$ & & $2_{-}$ & $K \geqslant M$ \\
$3_{ \pm}$ & $K \leqslant M \wedge K \leqslant-M$ & & $3_{-}$ & $K \leqslant M$ \\
$4_{ \pm}$ & $K \geqslant M \wedge K \leqslant-M$ & & $4_{-}$ & $K \geqslant M$ \\
\hline \hline
\end{tabular}

in the sense of Eq. (47), then one of the solutions $1_{+}, n=0, i=$ 0 and $3_{+}, n=0, i=0$ from Table VII,

$$
\hat{\psi}_{t, 0}(\theta)=e^{\sqrt{\zeta / B} \cos (\theta)} \sin ^{M-K}\left(\frac{\theta}{2}\right) \cos ^{ \pm(M+K)}\left(\frac{\theta}{2}\right),
$$

is not square integrable in any neighborhood of $\theta=\pi$. The connection between the boundedness of the effective centrifugal potential Eq. (45) and the square integrability of solutions to Eq. (44) can then be seen as follows: By applying Eq. (48) to the solutions stated in Eqs. (49) and (50), one finds that Eqs. (49) and (50) and their respective linearly independent second solutions are not both square integrable at $\theta=0$ or $\pi$ exactly when the centrifugal potential is confining at that end point. It is thus natural to expect that if the centrifugal potential at $\theta=0$ or $\pi$ remains bounded any solution to Eq. (44) is square integrable at that particular end point. Hence, if $M=K=0$ then any solution to Eq. (44) should be square integrable on the entire interval $(0, \pi)$.

We now introduce a terminology from Sturm-Liouville theory to make this observation precise: The operator $\hat{\mathcal{H}}_{t}$ is called limit-circle at $\theta=0$ or $\pi$ if and only if for one value $E_{t} \in \mathbb{C}$ both linearly independent solutions to $\hat{\mathcal{H}}_{t} \hat{\psi}_{t}=E_{t} \hat{\psi}_{t}$ are square integrable at $\theta=0$ or $\pi$, respectively. Otherwise, the operator $\hat{\mathcal{H}}_{t}$ is called limit-point at that end point.

The rigorous footing for our previous argument is the Weyl alternative [77] which states that the limit-circle property is independent of the energy $E_{t}$. Thus, if one verifies for a fixed energy $E_{t}$ that both solutions to Eq. (44) are square integrable at one of the end points, this will be the case for any other $E_{t} \in \mathbb{C}$ as well.

We summarize our preceding discussion by observing that $\hat{\mathcal{H}}_{t}$ has the following properties.

(1) $\hat{\mathcal{H}}_{t}$ is limit-circle at both end points if $M=K=0$, i.e., the centrifugal potential at both end points is bounded and all solutions to Eq. (44) are square integrable on the entire interval $(0, \pi)$.

(2) $\hat{\mathcal{H}}_{t}$ is limit-circle at $\theta=0$ and limit-point at $\theta=\pi$ if $M=K \neq 0$, i.e., the centrifugal potential is bounded at $\theta=0$, confining at $\theta=\pi$, and all solutions to Eq. (44) are square integrable close to $\theta=0$.

(3) $\hat{\mathcal{H}}_{t}$ is limit-circle at $\theta=\pi$ and limit-point at $\theta=0$ if $M=-K \neq 0$, i.e., the centrifugal potential is bounded at $\theta=$ $\pi$, confining at $\theta=0$, and all solutions to Eq. (44) are square integrable close to $\theta=\pi$.

(4) $\hat{\mathcal{H}}_{t}$ is limit-point at both end points in any other case, i.e., the centrifugal potential is confining at both end points. In this case, there do exist square integrable solutions but there do not exist two linearly independent solutions for a fixed energy that are both square integrable at the same end point.

Although we have obtained a rather complete description of when to expect normalizable or non-normalizable solutions, we still require a condition to exhibit the physical eigenstates among the normalizable ones. In fact, not every normalizable solution to Eq. (44) is also an eigenstate in general. This is only true when $\hat{\mathcal{H}}_{t}$ is a limit-point at both end points. If $\hat{\mathcal{H}}_{t}$ is a limit-circle at an end point, then a normalizable solution $\psi_{t}$ to Eq. (44) is an eigenfunction to $\hat{\mathcal{H}}_{t}$ if it satisfies at the limit-circle end points

$$
\lim _{\theta \rightarrow 0, \pi} \sin (\theta) \hat{\psi}_{t}^{\prime}(\theta)=0 .
$$

The physical interpretation of the condition in Eq. (51) is that eigenstates of the pendulum must have bounded wave functions and this condition filters out precisely the unbounded square integrable solutions. Mathematically, this condition is needed in order to obtain a self-adjoint operator $\hat{\mathcal{H}}_{t}$.

To illustrate our findings, we consider the limit-circle case at both end points $M=K=0$ and derive a solution using Eq. (48) that is not obtained by the quantum Hamilton-Jacobi theory. In this case, the operator introduced in Eq. (44) simplifies to

$$
\begin{aligned}
\hat{\mathcal{H}}_{t} \hat{\psi}_{t}(\theta)= & B\left(-\frac{d^{2}}{d \theta^{2}}-\cot \theta \frac{d}{d \theta}\right) \hat{\psi}_{t}(\theta) \\
& -\left(\eta \cos \theta+\zeta \cos ^{2} \theta\right) \hat{\psi}_{t}(\theta)=E_{t} \hat{\psi}_{t}(\theta) .
\end{aligned}
$$

Now, let $\eta= \pm 2 \sqrt{B} \sqrt{\zeta}$ and $E_{t}=-\xi$; then a solution to Eq. (52) is provided by the $1_{ \pm}, n=0$, and $i=0$ solution from Table VII, which reads

$$
\hat{\psi}_{t, 0}(\theta)=e^{ \pm \sqrt{\zeta / B} \cos (\theta)} .
$$

Applying the formula in Eq. (48) to this solution yields then another solution to Eq. (52) for the same energy value that is not contained in Table VII:

$$
\begin{aligned}
\hat{\varphi}_{t, 0}(\theta):= & \frac{e^{ \pm \sqrt{\frac{\zeta}{B}}(\cos (\theta)-2)}}{2}\left[\operatorname{Ei}\left( \pm 4 \sqrt{\frac{\zeta}{B}} \sin ^{2}\left(\frac{\theta}{2}\right)\right)\right. \\
& \left.-e^{ \pm 4 \sqrt{\frac{\zeta}{B}}} \operatorname{Ei}\left(\mp 4 \sqrt{\frac{\zeta}{B}} \cos ^{2}\left(\frac{\theta}{2}\right)\right)\right]
\end{aligned}
$$

where $\mathrm{Ei}$ is the exponential integral function. In other words, $\hat{\varphi}_{t, 0}$ is a square integrable solution to Eq. (52) that has not been found by the quantum Hamilton-Jacobi theory, which does not satisfy the condition in Eq. (51), i.e.,

$$
\lim _{\theta \rightarrow 0, \pi} \sin (\theta) \hat{\varphi}_{t, 0}^{\prime}(\theta) \neq 0,
$$

and is therefore not an eigenstate of the pendulum.

\section{Hyperbolic top}

After the comprehensive treatment of the trigonometric equation (10), we just state the results for the hyperbolic potential $(15)$ on $(0, \infty)$ for $\zeta>0$. In this case, the centrifugal potential is

$$
V_{\text {cent,h }}(\theta)=-\left(M^{2}+K^{2}\right) \operatorname{csch}^{2}(\theta)+2 M K \operatorname{coth}(\theta) \operatorname{csch}(\theta) .
$$



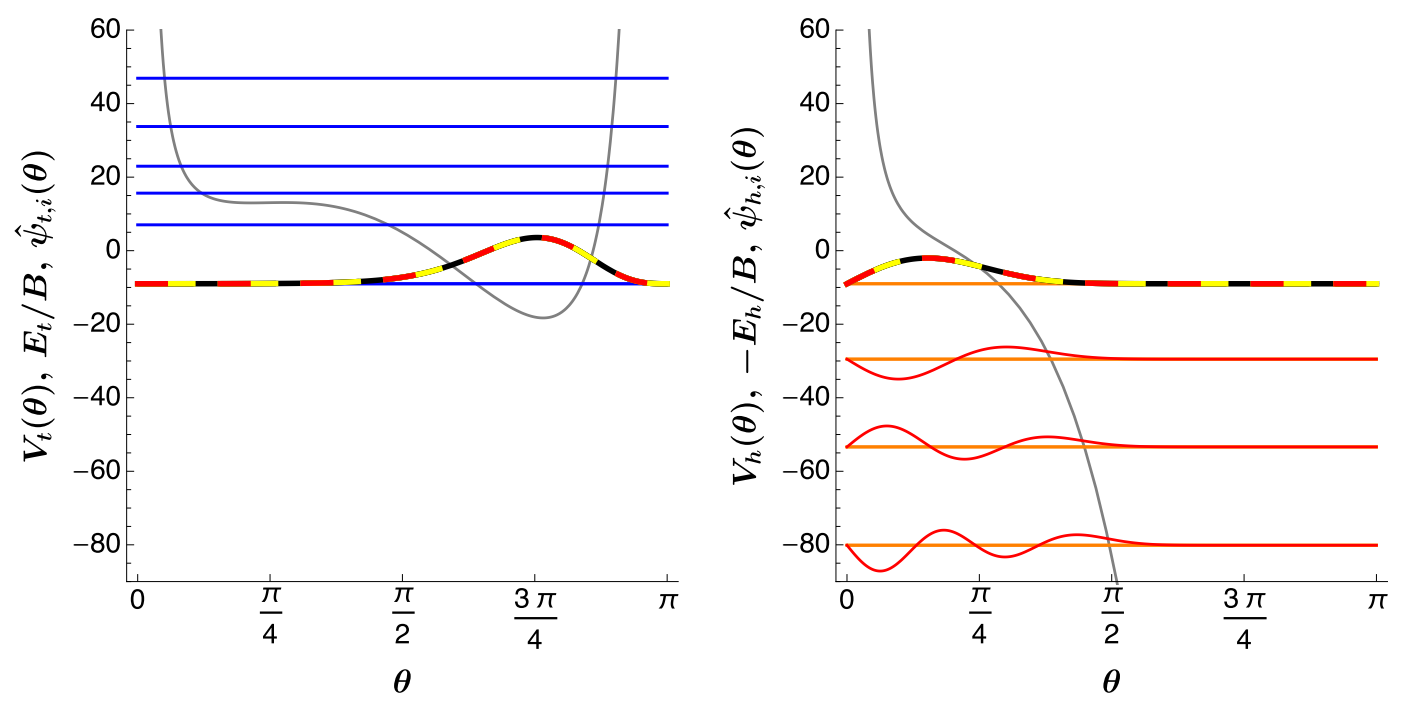

FIG. 6. Normalizable closed-form sample wave functons $\hat{\psi}_{t, i}(\theta)$ (left) and $\hat{\psi}_{h, i}(\theta)$ (right). Black, sector $1_{ \pm}$; yellow, sector $2_{ \pm}$; red, sector $3_{ \pm}$. Blue: numerical eigenenergies for the trigonometric top (left). Orange: numerical eigenenergies for the hyperbolic top (right). Potentials are shown in gray. Both plots: $M=2, K=1, \zeta=25, \eta=-30$. Color coding is the same as in Fig. 2.

Since the hyperbolic potential converges to $\infty$ as $\theta \rightarrow \infty$, this already implies that $\hat{\mathcal{H}}_{h}$ is a limit-point at $\theta=\infty$. Thus, there is at most one square integrable solution for any energy value to $\hat{\mathcal{H}}_{h}$ at $\theta=\infty$ and it suffices to study the boundary value $\theta=0$. For $\theta=0$, the centrifugal potential $V_{\text {cent,h }}$ is bounded only if $K=M$. The boundedness of the centrifugal potential is then, as for the trigonometric potential, equivalent to $\hat{\mathcal{H}}_{h}$ being a limit-circle. Thus, exactly when $K=M$ all solutions to Eq. (15) for arbitrary energies $E$ are square integrable at $\theta=0$. Similar to the boundary condition in Eq. (51) for the limit-circle case with trigonometric potential, we get an analogous boundary condition in the limit-circle case at $\theta=0$ for the hyperbolic potential, filtering out the physical eigenstates among the square integrable solutions:

$$
\lim _{\theta \rightarrow 0} \sinh (\theta) \hat{\psi}_{h}^{\prime}(\theta)=0 .
$$

A second linearly independent solution $\hat{\varphi}_{h}$ to some given solution $\hat{\psi}_{h}$ for the same energy can be obtained from

$$
\hat{\varphi}_{h}(\theta)=\hat{\psi}_{h}(\theta) \int_{1}^{\theta} \frac{d t}{\sinh (t) \hat{\psi}_{h}(t)^{2}} .
$$

\section{EXAMPLES AND OBSERVATIONS}

In this section we take a closer look at the spectral patterns in the cases $(K, M)=(1,2),(1,1),(0,1)$, and $(0,1 / 2)$ and retrieve the symmetry-based special-case classifications of closed-form solutions from our earlier work [33,61] for the spherical pendulum, i.e., for $K=0$, as well as the planar pendulum and the Razavy system, i.e., for $(K, M)=(0,1 / 2)$.
In Figs. 2-5, we use the following color coding and graphical symbols: blue and orange curves show numerical eigenenergies of the trigonometric and hyperbolic top obtained via the analytic matrix elements of Appendix B and overlap matrices of Appendix C, respectively. The algebraic energies of sectors $1_{ \pm}, 2_{ \pm}, 3_{ \pm}$, and $4_{ \pm}$pertaining to normalizable wave functions are represented by black, yellow, red, and green disks for $n \leqslant 3$. Nested disks represent eigenenergies of coinciding closed-form solutions for different sectors. If the closed-form wave functions are not normalizable, the corresponding disks are furnished with a gray cross. The extrema of the potentialenergy curves of the trigonometric top are shown by gray curves. Note that level crossings (genuine and avoided) are marked by the (integer) values of the topological index $\kappa=$ $\frac{\eta}{\sqrt{B \zeta}}$, which can be used as their label.

In Figs. 6 and 7 we show algebraic wave functions colored according to same the scheme as described above for Figs. 2-5. Likewise, the numerical eigenenergies of the trigonometric (left) and hyperbolic top (right) are again shown in blue and orange, respectively. Gray curves show the extrema of the trigonometric and hyperbolic top potentials.

As for the total number of normalizable closed-form solutions obtained (for $n \leqslant 3$ ), we see in Figs. 2-4 as well as in Table VII that for the trigonometric top it is

$$
\#_{t}\{\text { algebraic solutions }\}=\left(n_{\max }+1\right)\left(n_{\max }+2\right) \text {. }
$$

Note, that the number of solutions takes into account all sectors for both branches $\pm \sqrt{\zeta}$. In the hyperbolic case we have

$$
\#_{h}\{\text { algebraic solutions }\}=\left\{\begin{array}{l}
\left(n_{\max }+1\right)\left(n_{\max }+2\right)-\frac{\left(n_{\max }-|M+K|+1\right)\left(n_{\max }-|M+K|+2\right)}{2} \text { for } n_{\max }-|M+K| \geqslant 0 \\
\left(n_{\max }+1\right)\left(n_{\max }+2\right) \text { for } n_{\max }-|M+K|<0
\end{array} .\right.
$$



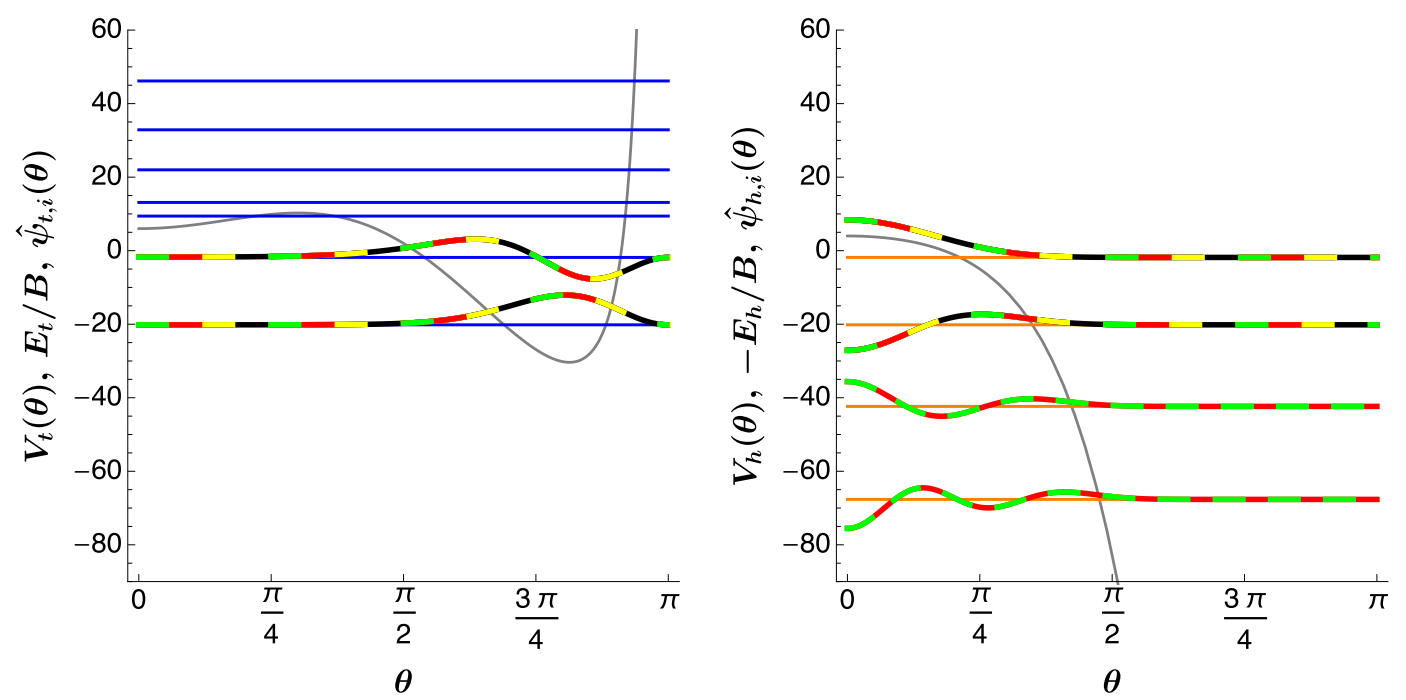

FIG. 7. Normalizable closed-form sample wave functons $\hat{\psi}_{t, i}(\theta)$ (left) and $\hat{\psi}_{h, i}(\theta)$ (right). Black, sector $1_{ \pm}$; yellow, sector $2_{ \pm}$; red, sector $3_{ \pm}$; green, sector $4_{ \pm}$. Blue: numerical eigenenergies for the trigonometric top (left). Orange: numerical eigenenergies for the hyperbolic top (right). Potentials are shown in gray. Both plots: $M=1, K=1, \zeta=25, \eta=-30$. Color coding is the same as in Fig. 3.

Here, $K, M \in \mathbb{Z}$ and $n_{\max }$ is the highest $n$ for which one can derive closed-form solutions. As explained in Sec. IIIE, we choose $n_{\max }=3$, which always yields 20 closed-form solutions for each configuration $(K, M, \eta, \zeta)$ in the trigonometric top case for either of the two branches $\pm \sqrt{\zeta}$. On the other hand, for the hyperbolic top with $(M, K)=(0,0),(1,1)$, or $(2,1)$, we find 10,17 , or 19 different closed-form solutions, whereas for $4-|M+K|>0$ the total number becomes 30 for each $(K, M, \eta, \zeta)$.

\section{A. $K=1, M=2$}

We can read off from Fig. 2 that there are ten normalizable algebraic solutions for each of the sectors $1_{ \pm}$(black). Out of these, sector $1_{+}(\kappa \geqslant 6)$ contains only eigenvalues of the trigonometric top, whereas the eigenvalues of the trigonometric and hyperbolic top coincide for sector $1_{-}(\kappa \leqslant$ $6)$. Furthermore, we have six normalizable and four nonnormalizable closed-form solutions for sectors $2_{+}(\kappa \geqslant 4)$ and $2_{-}(\kappa \leqslant 4)$ each, shown in yellow. As all the normalizable solutions coincide with sector $1_{ \pm}$solutions for $n \leqslant 2$, the relative distribution of the solutions over the trigonometric and hyperbolic top case is identical to that of sector $1_{ \pm}$solutions.

Sector $3_{+}$(red) contains 1 normalizable solution at $(\kappa, n)=$ $(6,3)$ of the trigonometric top, which is identical to the normalizable solution of sector $1_{+}$at $(\kappa, n)=(6,0)$. A similar situation occurs for the highest excited sector $3_{-}$(red) solution at $(\kappa, n)=(-6,3)$ in that it coincides with the sector $1_{-}$ solution at $(\kappa, n)=(6,0)$. While all other solutions of sector $3_{+}$are non-normalizable for the trigonometric and hyperbolic top, every other sector 3 - solution is normalizable for the hyperbolic top. There exist six normalizable sector $4_{-}$(green) solutions $(\kappa \leqslant 1)$, overlapping with sector 3 _ solutions, one for $n \leqslant 2$. All other sector $4_{+}$and $4_{-}$(green) solutions are non-normalizable.

Thus we see that for all configurations with $K$ or $M \neq 0$ there emerge three regions of normalizable solutions, which may partly overlap: first, one for the $\sqrt{\zeta}$ branch, which pertains to the trigonometric top only; second, one for the $-\sqrt{\zeta}$ branch, which pertains to the hyperbolic top only; and, third, one for the $-\sqrt{\zeta}$ branch that pertains to both the trigonometric and hyperbolic top. Ultimately, the cardinality of the overlap of the second and third region $(-\sqrt{\zeta}$ branch) as well as the spectral gap of non-normalizability to the first region of the $\sqrt{\zeta}$ branch suggest a vectorial measure for the distribution of these regions between the trigonometric and hyperbolic numerical spectra, namely, $|K+M|$ and $|K-M|$. The former provides the number of the highest algebraic eigenenergies of the hyperbolic top which are not coincident with any of the trigonometric top eigenenergies. The latter gives the number of non-normalizable solutions in between the regions of branch $\sqrt{\zeta}$ and $-\sqrt{\zeta}$, i.e., the above-mentioned spectral gap denoted by gray crosses and running diagonally from the top left to the bottom right. As shown below for the configurations $(K, M)=(1,1),(1,0)$, this measure is valid for all $K, M \in \mathbb{Z}$.

These observations are consistent with Eqs. (59) and (60): the overall number of normalizable algebraic solutions is 20 for the trigonometric top and 19 for the hyperbolic one. Additionally, there are 20 non-normalizable solutions for either case.

\section{B. $K=1, M=1$}

The corresponding eigenenergies are shown in Fig. 3 and a sampling of the eigenfunctions in Fig. 7. There are 20 closed-form solutions for the trigonometric and 17 for the hyperbolic top, in agreement with Eqs. (59) and (60), respectively. In addition, we found seven non-normalizable closed-form solutions for the symmetric top.

$$
\text { C. } K=0
$$

For the trigonometric top with $n=0$ and $\kappa=2 k$, the case of $K=0$ coincides with that of the spherical pendulum, as investigated via SUSY QM in our previous work [33,59,60,65]. The closed-form solutions listed in Ref. [33] can now be directly retrieved from Table VII. Table IX provides their 
TABLE IX. Correlations between sectors and the algebraic seedstate cases obtained from SUSY QM in [33] for the spherical pendulum $(K=0)$. Note that in our earlier work the seed states $\hat{\psi}_{t, 0}(\theta)$ were presented in gauged form, $\psi_{t, 0}(\theta)=\hat{\psi}_{t, 0}(\theta) \sqrt{\sin \theta}$ [see Eq. (11)].

\begin{tabular}{lccc}
\hline \hline Sector & Case & $\kappa$ & $\psi_{t, 0}(\theta)$ \\
\hline $1_{+}$ & $1_{-}$ & $2(M+1)$ & $(\sin \theta)^{M+\frac{1}{2}} \mathrm{e}^{\sqrt{\zeta / B} \cos \theta}$ \\
$2_{+}$ & $2_{+}$ & 2 & $(\sin \theta)^{\frac{1}{2}}(\tan \theta)^{-M} \mathrm{e}^{\sqrt{\zeta / B} \cos \theta}$ \\
$3_{+}$ & $2_{-}$ & 2 & $(\sin \theta)^{\frac{1}{2}}(\tan \theta)^{M} \mathrm{e}^{\sqrt{\zeta / B} \cos \theta}$ \\
$4_{+}$ & $1_{+}$ & $2(1-M)$ & $(\sin \theta)^{-M+\frac{1}{2}} \mathrm{e}^{\sqrt{\zeta / B} \cos \theta}$ \\
\hline \hline
\end{tabular}

summary as well as a correlation between the algebraic sectors and the seed-state cases introduced in Ref. [33].

Figure 4 displays the spectral pattern for $(K, M)=(0,1)$ as the choice example. We find again that, in agreement with Eqs. (59) and (60), there are 20 normalizable closed-form solutions for the trigonometric and 14 for the hyperbolic top. Note that for $n=0$ only the sector $1_{+}$solution $(\kappa=4)$ is normalizable. Compared with our previous SUSY QM work as summarized in Ref. [33], the present paper based on the QHJ theory delivers ten times as many closed-form solutions (for $M>0)$.

$$
\text { D. } K=0, M=1 / 2
$$

The nonphysical choice of $M=1 / 2$ for a symmetric top makes it possible to retrieve all our previous results concerning the quasisolvability of the planar pendulum (in the trigonometric case) and the Razavy system (in the hyperbolic case), as reported in Refs. [32,61].

Our previous work [32,61] examined the spatial symmetry classes and irreducible representations $\Gamma_{t, h}$ of both the planar pendulum and the Razavy systems. Table $\mathrm{X}$ lists the closed-form solutions for $(K, M)=(0,1 / 2)$ as retrieved from Table VII for $n \geqslant 0$ and provides a correlation between the algebraic sectors and the irreducible representations of Ref. [61]. Note that in the pendulum case the domain of the polar angle $\theta$ becomes $[0,2 \pi)$.

The energy levels of the planar pendulum and the Razavy systems together with the classification of the spectral patterns according to their algebraic sectors are shown in Fig. 5. Compared with our previous work, the figure includes negative

TABLE X. Planar pendulum (left) and the Razavy system (right) as special cases of the trigonometric and hyperbolic tops, respectively, for $(K, M)=(0,1 / 2)$ : correlations between algebraic sectors and the irreducible representation $\Gamma_{t, h}$ of symmetry groups from Ref. [61]. Note that in our earlier work the seed functions $\hat{\psi}_{t, 0}(\theta)$ were presented in gauged form, $\psi_{t, 0}(\theta)=\hat{\psi}_{t, 0}(\theta) \sqrt{\sin \theta}$ [see Eq. (11)].

\begin{tabular}{|c|c|c|c|c|c|c|c|}
\hline \multicolumn{4}{|c|}{ Planar pendulum } & \multicolumn{4}{|c|}{ Razavy system } \\
\hline Sector & $\Gamma_{t}$ & $\kappa$ & $\psi_{t, 0}(\theta)$ & Sector & $\Gamma_{h}$ & $\kappa$ & $\psi_{h, 0}(\theta)$ \\
\hline $1_{+}$ & $A_{2}$ & 3 & $\sin \theta \mathrm{e}^{\sqrt{\zeta / B} \cos \theta}$ & 3 & $A^{\prime \prime}$ & 2 & $\sinh \frac{\theta}{2} \mathrm{e}^{-\sqrt{\zeta / B} \cosh \theta}$ \\
\hline $2_{+}$ & $B_{1}$ & 2 & $\cos \frac{\theta}{2} \mathrm{e}^{\sqrt{\zeta / B} \cos \theta}$ & & & & \\
\hline $3_{+}$ & $B_{2}$ & 2 & $\sin \frac{\theta}{2} \mathrm{e}^{\sqrt{\zeta / B} \cos \theta}$ & $4_{-}$ & $A^{\prime}$ & 1 & $\mathrm{e}^{-\sqrt{\zeta / B} \cosh \theta}$ \\
\hline $4_{+}$ & $A_{1}$ & 1 & $\mathrm{e}^{\sqrt{\zeta / B} \cos \theta}$ & & & & \\
\hline
\end{tabular}

$\eta$ values. Since for $(K, M)=(0,1 / 2)$ the topological index $\kappa$ can take both odd and even integer values, one has to multiply the number of solutions as given by Eq. (59) by a factor of 4. Thus, for the planar pendulum, there are 40 normalizable closed-form solutions in the $\sqrt{\zeta}$ and $-\sqrt{\zeta}$ branch each and 40 normalizable closed-form solutions for the Razavy system.

\section{CONCLUSIONS}

We have examined conditional quasisolvability of a symmetric top subject to collinear orienting and aligning interactions - the symmetric top quantum pendulum. We did so by invoking the quantum Hamilton-Jacobi theory. Although the symmetric top quantum pendulum represents a considerable generalization of the planar and spherical pendula, we have been able not only to retrieve the closed-form solutions identified previously for the special planar [61] and spherical $[33,59,60]$ cases but also to find a number of solutions, including those for the generalized symmetric top case. For the spherical pendulum case alone we have found ten times as many solutions as were known from our previous work based on supersymmetric quantum mechanics.

Our previous work within the framework of SUSY QM required an educated guess for the superpotential. A complexified superpotential makes also an appearance in the QHJ theory - as the quantum momentum function. However, QHJ provides a recipe for constructing the QMF, although it still needs an educated guess concerning the choice of suitable coordinates for expressing the closed-form solutions. This latter task is much less ad hoc than the former one and therefore easier to fulfill.

Apart from the trigonometric symmetric top, we have also tackled its anti-isospectral counterpart, the hyperbolic top, and examined the patterns of the algebraic and numerical spectra of both types of top for special choices of the projection quantum numbers $K$ and $M$. We found that the distribution patterns of the algebraic spectra from the different algebraic sectors can be characterized by the quantum number measures $|K+M|$ and $|K-M|$ (see Figs. 2-4). An inspection of these figures reveals that the general pattern of the $4+4$ triangles (one for each of the four algebraic sectors or colors and type of top) is independent of $K$ and $M$. However, the triangles are shifted with respect to each other, with the shift depending on both $|K-M|$ and $|K+M|$. These shifts in fact govern how many and which solutions coincide, thus determining the total number of independent solutions. Moreover, as illustrated by the said figures, these shifts are characteristic for the transformation from the symmetric to the spherical to the planar top. Note that in none of Figs. 2-4 are there more than four normalizable solutions at a given $\kappa$. But for the special case of $M=1 / 2$, there are up to eight solutions at a given $\kappa$ (see Fig. 5).

In order to evaluate the physical relevance (i.e., normalizability) of the solutions found, we applied the limit-point and limit-circle classification and found a condition that identifies bounded square integrable solutions.

As noted in our previous work, the topological index $\kappa=\frac{\eta}{\sqrt{B \zeta}}$ labels the genuine and avoided crossings of the eigenenergy levels. According to, e.g., Eq. (34) and Table III, the $\eta$ spacing between the level crossings, i.e., $2 \sqrt{\zeta}$ for the 
$K, M$ integer and $\sqrt{\zeta}$ for the $K, M$ half-integer, is identical to the boundary at infinity of the rationalized QMF $\tilde{p}_{f}(z)$ on the complexified domain $\mathcal{D}_{c}(z)$ and, respectively, to the boundary at zero of the likewise rationalized $\mathrm{QMF} \hat{p}_{f}(w)$ on $\mathcal{D}_{c}(w)$ with the mapping $z \mapsto w=1 / z$ between the domains. Like the quasisolvability conditions, this boundary stems from the domain compactification described in Sec. III B. It would be interesting to see whether this is a general feature of all periodic Hamiltonians and their "dual" anti-isospectral hyperbolic partners. Of interest is also the question of whether one could find any rules for determining the number of accessible exact solutions for $n_{\max }>3$.

The spectral properties at integer values of $\kappa$-and hence the quasiexact solvability-may be difficult to verify experimentally, mainly due to the uncertainties in setting the strengths of the electrostatic $(\eta)$ and radiative $(\zeta)$ fields. The interaction parameters for representative molecules are listed in Table I of Ref. [65] while Table II of Ref. [65] provides the conversion factors needed to obtain the dimensionless reduced parameters from the molecular parameters expressed in customary units.

However, there are other features in the numerically calculated spectra of Figs. 2-5 that may be amenable to experimental testing. While all energy levels of the hyperbolic top are monotonous functions of $\eta$, the lower curves for the trigonometric top exhibit extrema. These become more numerous - and also arise for the higher-lying levels - at larger values of $\zeta$. While these extrema are less pronounced for $K \neq 0$ (see Figs. 2 and 3), they become more conspicuous in the spherical pendulum case (see Fig. 4). As detailed in our previous work $[33,65]$, the loci of the extrema, where all states energetically higher than the analytic solutions exhibit avoided crossings for a large-enough $\zeta$, coincide with the conditions of quasisolvability (even integer values of $\kappa$ ). A similar situation arises for the planar pendulum, Fig. 5, where again the conditions of quasisolvability (integer values of $\kappa$ ) coincide with the loci of genuine (odd $\kappa$ ) or avoided (even $\kappa$ ) intersections of the higher states; note that the latter occur only for a large-enough $\zeta[32,61]$.

The spectra of the spherical and planar pendula are always such that the bottom part of the spectra for the values of $\kappa$ fulfilling the QS condition consists of single states whereas all higher states are found to form genuine or avoided crossings for large $\zeta$. This general pattern could in principle be confirmed in spectroscopic experiments-by scanning the $\eta$ or $\zeta$ parameters of our trigonometric model Hamiltonian, Eq. (10).

We note that the algebraic solutions found may serve as benchmarks for numerical analysis and the polynomial ansatz they suggest could be useful for numerical calculations beyond quasisolvability, i.e., for noninteger values of the topological index $\kappa$.

In our ongoing work, we deal with the case of symmetric tops subject to noncollinear orienting and aligning interactions-a much harder problem, both analytically and numerically.

\section{ACKNOWLEDGMENT}

Support by the Deutsche Forschungsgemeinschaft (DFG) through Grants No. SCHM 1202/3-1 and No. FR 3319/3-1 is gratefully acknowledged.

\section{APPENDIX A: QUANTUM HAMILTON-JACOBI EQUATION IN RATIONAL FORM}

In order to be able to make use of the Laurent series expansion and the residue calculus for solving the quantum Hamilton-Jacobi equation (17), we need to ensure that all terms besides those which contain the quantum momentum function are rational.

To this end, we first transform the variable $\theta$ into a new variable $z=z(\theta)$ such that only rational terms in the new variable remain. The sought transformation is induced by the mapping (see Ref. [68])

$$
p(\theta)=-\left.\frac{i}{\theta^{\prime}(z)}\left[\tilde{p}(z)+\frac{1}{2} \sqrt{B} \ln \left(\theta^{\prime}(z)\right)^{\prime}\right]\right|_{z=z(\theta)}
$$

of the new meromorphic quantum momentum function $\tilde{p}(z)$ and leads to

$$
\begin{gathered}
\tilde{p}(z)^{2}+\sqrt{B} \tilde{p}^{\prime}(z)+\frac{1}{2} B\left[\frac{\theta^{\prime \prime \prime}(z)}{\theta^{\prime}(z)}-\frac{3}{2} \frac{\theta^{\prime \prime}(z)^{2}}{\theta^{\prime}(z)^{2}}\right] \\
+\theta^{\prime}(z)^{2}[E-V(\theta(z))]=0,
\end{gathered}
$$

which can be viewed as a normal form [68] of the Riccati equation (17) with the inverse transformation of coordinates 8 $\theta=\theta(z)$.

Equation (A2) can be recast in terms of the Schwarzian derivative,

$$
\mathrm{S}(\theta)(z)=\left(\frac{\theta^{\prime \prime}(z)}{\theta^{\prime}(z)}\right)^{\prime}-\frac{1}{2}\left(\frac{\theta^{\prime \prime}(z)}{\theta^{\prime}(z)}\right)^{2},
$$

with the result

$$
\tilde{p}(z)^{2}+\sqrt{B} \tilde{p}^{\prime}(z)+\frac{1}{2} B \mathrm{~S}(\theta)(z)+\theta^{\prime}(z)^{2}[E-V(\theta(z))]=0 .
$$

According to, e.g., Refs. [69,78], all coordinate transformations $z=z(\theta)$ are admissible for which $r(y)$ in

$$
\theta(z)=\int^{z} \frac{d y}{\sqrt{r(y)}}
$$

are polynomials in $y$ of degree smaller than or equal to 4 . Thus all terms $\theta^{\prime}(z)^{2}$ and $\theta^{(n)}(z) / \theta^{(m)}(z)$, with $n$ and $m$ the orders of the derivatives, must be rational functions, including $\mathrm{S}(\theta)(z)$. By ensuring that also $V_{t}(\theta(z))$ is a rational function via a suitable choice of $z(\theta)$, we obtain rational solutions $\tilde{p}(z)$ of Eq. (A4).

We note that any Möbius transformation $\frac{\alpha z(\theta)+\beta}{\gamma z(\theta)+\delta}$ of the new coordinate $z$ will leave the terms in Eq. (A4) rational, since the Schwarzian derivative is invariant under Möbius transformations.

By making the ansatz

$$
\tilde{\psi}(z)=e^{\frac{1}{\sqrt{B}} \int^{z} \tilde{p}(y) d y}
$$

\footnotetext{
${ }^{8}$ Multivaluedness, if existing, has to be taken into account, unless it only arises from a phase shift of the form $e^{i \pi v}$ with the $v$ integer.
} 
for the wave function of the transformed problem, we can derive from Eq. (A4) a new Schrödinger-type equation:

$$
-B \frac{1}{\theta^{\prime}(z)^{2}} \tilde{\psi}^{\prime \prime}(z)+\left[-\frac{1}{2} B \frac{\mathrm{S}(\theta)(z)}{\theta^{\prime}(z)^{2}}+V(\theta(z))\right] \tilde{\psi}(z)=E \tilde{\psi}(z) .
$$

The old wave function, $\psi(\theta)$, is then related to the new one, $\tilde{\psi}(z)$, via

$$
\psi(\theta)=\left.\tilde{\psi}(z) \sqrt{\theta^{\prime}(z)}\right|_{z=z(\theta)},
$$

which is equivalent to Eq. (A1) in that it induces the transformation from the original Schrödinger equation (12) to the new equation. Equation (A7) with purely rational terms like Eq. (A1) induces the transformation from the original QHJ equation (17) to its normal Riccati form Eq. (A4).

By identifying the rational function

$$
\tilde{V}(z)=V(\theta(z))-\frac{1}{2} B \frac{\mathrm{S}(\theta)(z)}{\theta^{\prime}(z)^{2}}
$$

as the new potential, we can recast Eq. (A4) in a compact, rational form:

$$
\tilde{p}(z)^{2}+\sqrt{B} \tilde{p}^{\prime}(z)+\theta^{\prime}(z)^{2}[E-\tilde{V}(\theta(z))]=0 .
$$

\section{APPENDIX B: MATRIX ELEMENTS FOR DETERMINING THE NUMERICAL SPECTRA OF THE TRIGONOMETRIC TOP}

The numerical spectra in Figs. 3-5 were determined by diagonalizing the matrix representation of Hamiltonian (1) in the symmetric top basis set

$$
|J K M\rangle=(-1)^{M-K} \sqrt{\frac{2 J+1}{8 \pi^{2}}} D_{-M-K}^{J}
$$

with $D_{M K}^{J}$ the Wigner $D$ matrices [2]. Note that $J \geqslant$ $\max \{K, M\}$.

The potential-energy terms, Eq. (2), can be recast in terms of the Wigner $D$ matrices as

$$
\cos \theta=D_{00}^{1}
$$

and

$$
\cos ^{2} \theta=\frac{2}{3} D_{00}^{2}+\frac{1}{3} D_{00}^{0}
$$

The matrix elements of the $\cos \theta$ and $\cos ^{2} \theta$ operators, Eqs. (B2) and (B3), were obtained via the Gaunt integral in terms of the $3-j$ symbols

$$
\begin{aligned}
\int_{0}^{2 \pi} & \int_{0}^{\pi} \int_{0}^{2 \pi} D_{M_{3} K_{3}}^{J_{3}}(\varphi, \theta, \chi) D_{M_{2} K_{2}}^{J_{2}}(\varphi, \theta, \chi) D_{M_{1} K_{1}}^{J_{1}} \\
& \times(\varphi, \theta, \chi) d \phi \sin \theta d \theta d \chi \\
& =8 \pi^{2}\left(\begin{array}{ccc}
J_{1} & J_{2} & J_{3} \\
M_{1} & M_{2} & M_{3}
\end{array}\right)\left(\begin{array}{lll}
J_{1} & J_{2} & J_{3} \\
K_{1} & K_{2} & K_{3}
\end{array}\right)
\end{aligned}
$$

with the result

$$
\begin{aligned}
& \left\langle J^{\prime} K^{\prime} M^{\prime}|\cos \theta| J K M\right\rangle \\
& =\sum_{j=-1}^{1}(-1)^{M-K} \frac{\sqrt{(2 J+1)(2(J+j)+1)}}{8 \pi^{2}}
\end{aligned}
$$

$$
\begin{aligned}
& \times\left(\begin{array}{ccc}
J & 1 & J+j \\
-M & 0 & M
\end{array}\right)\left(\begin{array}{ccc}
J & 1 & J+j \\
-K & 0 & K
\end{array}\right) \\
& \times \delta_{J^{\prime},(J+j)} \delta_{M^{\prime}, M} \delta_{K^{\prime}, K}
\end{aligned}
$$

and

$$
\begin{aligned}
& \left\langle J^{\prime} K^{\prime} M^{\prime}\left|\cos ^{2} \theta\right| J K M\right\rangle \\
& =\sum_{j=-2}^{2}(-1)^{M-K} \frac{\sqrt{(2 J+1)(2(J+j)+1)}}{8 \pi^{2}} \\
& \times\left[\frac{2}{3}\left(\begin{array}{ccc}
J & 2 & J+j \\
-M & 0 & M
\end{array}\right)\left(\begin{array}{ccc}
J & 2 & J+j \\
-K & 0 & K
\end{array}\right)\right. \\
& \left.+\frac{1}{3}\left(\begin{array}{ccc}
J & 0 & J+j \\
-M & 0 & M
\end{array}\right)\left(\begin{array}{ccc}
J & 0 & J+j \\
-K & 0 & K
\end{array}\right)\right] \\
& \times \delta_{J^{\prime},(J+j)} \delta_{M^{\prime}, M} \delta_{K^{\prime}, K} .
\end{aligned}
$$

Ultimately, the matrix elements of Hamiltonian $\mathcal{H}$ of Eq. (1) take the analytic form

$$
\begin{aligned}
\left\langle J^{\prime} K^{\prime} M^{\prime}|\mathcal{H}| J K M\right\rangle= & B\left(J(J+1)+\rho K^{2}\right) \delta_{J^{\prime}, J} \delta_{M^{\prime}, M} \delta_{K^{\prime}, K} \\
& -\eta\left\langle J^{\prime} K^{\prime} M^{\prime}|\cos \theta| J K M\right\rangle \\
& -\zeta\left\langle J^{\prime} K^{\prime} M^{\prime}\left|\cos ^{2} \theta\right| J K M\right\rangle . \quad(\mathrm{B} 7)
\end{aligned}
$$

\section{APPENDIX C: CALCULATION OF THE NUMERICAL SPECTRA OF THE HYPERBOLIC TOP}

For the numerical evaluation of the spectrum of the hyperbolic Hamiltonian of Eq. (15) on $(0, \infty)$ for $K=M=1$, a rapidly decaying sequence of basis functions is desirable. We circumvent this by making the substitution $x=\cosh (\theta)$ that leads to an equation on $(1, \infty)$ and a more robust numerical approximation of the spectrum.

It turned out to be advantageous to choose a set of linearly independent trial functions $\varphi_{i}(x):=\frac{e^{-\sqrt{\zeta} x}}{1+x} x^{i}$ the exponential decay of which coincided with the one of the known eigenfunctions. With these, we were able to construct a symmetric matrix approximation $H_{h}^{N}$ of the Hamiltonian by choosing a finite cutoff $N \in \mathbb{N}$ such that for $i, j \in\{1, \ldots, N\}$ the matrix elements read

$$
\left\langle i\left|H_{h}^{N}\right| j\right\rangle=\int_{0}^{\infty} \overline{\varphi_{i}(\cosh (\theta))} H_{h} \varphi_{j}(\cosh (\theta)) \sinh (\theta) d \theta .
$$

Such matrix elements can be computed using standard numerical integrators. Due to our particular choice of basis functions, the singularity of the potential at $\theta=0$ was removed and the integral was evaluated over smooth functions only. The price to pay was that the functions $\varphi_{i}$ do not form an orthonormal basis. Thus, an additional symmetric positive-definite overlap matrix $O$ with matrix elements

$$
\langle i|O| j\rangle=\int_{1}^{\infty} \overline{\varphi_{i}(x)} \varphi_{j}(x) d x
$$

had to be computed numerically. The energies of the hyperbolic pendulum were then obtained as the eigenvalues of the generalized problem

$$
H_{h}^{N} v=\lambda O v,
$$

which can be solved with a standard software package. 
[1] F. Aoiz, B. Friedrich, V. Herrero, V. S. Rabanos, and J. Verdasco, Chem. Phys. Lett. 289, 132 (1998).

[2] M. Härtelt and B. Friedrich, J. Chem. Phys. 128, 224313 (2008).

[3] B. Friedrich and D. Herschbach, J. Phys. Chem. A 103, 10280 (1999).

[4] M. Lemeshko, R. V. Krems, J. M. Doyle, and S. Kais, Mol. Phys. 111, 1648 (2013).

[5] F. Cooper, A. Khare, and U. Sukhatme, Phys. Rep. 251, 267 (1995).

[6] P. Brooks, Science 193, 11 (1976).

[7] H. J. Loesch and A. Remscheid, J. Chem. Phys. 93, 4779 (1990).

[8] B. Friedrich and D. Herschbach, Nature (London) 353, 412 (1991).

[9] J. Ortigoso, M. Rodríguez, M. Gupta, and B. Friedrich, J. Chem. Phys. 110, 3870 (1999).

[10] T. Seideman, Phys. Rev. Lett. 83, 4971 (1999).

[11] J. J. Larsen, K. Hald, N. Bjerre, H. Stapelfeldt, and T. Seideman, Phys. Rev. Lett. 85, 2470 (2000).

[12] L. Cai and B. Friedrich, Coll. Czech Chem. Commun. 66, 991 (2001).

[13] I. S. Averbukh and R. Arvieu, Phys. Rev. Lett. 87, 163601 (2001).

[14] M. Leibscher, I. S. Averbukh, and H. Rabitz, Phys. Rev. Lett. 90, 213001 (2003).

[15] M. Leibscher, I. S. Averbukh, and H. Rabitz, Phys. Rev. A 69, 013402 (2004).

[16] J. Toennies, Z. Phys. 177, 84 (1964).

[17] S. Stolte, Berichte Bunsen. Ges. Phys. Chem. 413, 84 (1982).

[18] H. Stapelfeldt, H. Sakai, E. Constant, and P. Corkum, Phys. Rev. Lett. 79, 2787 (1997).

[19] L. Y. Kim, J. H. Lee, H. A. Kim, S. K. Kwak, B. Friedrich, and B. S. Zhao, Phys. Rev. A 94, 013428 (2016).

[20] S. Truppe, H. Williams, M. Hambach, L. Caldwell, N. Fitch, E. Hinds, B. Sauer, and M. Tarbutt, Nature Physics 13, 1173 (2017).

[21] R. Bernstein, D. Herschbach, and R. Levine, J. Phys. Chem. 91, 5365 (1987).

[22] R. Krems, W. C. Stwalley, and B. Friedrich, Cold Molecules: Theory, Experiment, Applications (CRC, Boca Raton, FL, 2009).

[23] O. Dulieu and A. Osterwalder, Cold Chemistry: Molecular Scattering and Reactivity Near Absolute Zero (Royal Society of Chemistry, Cambridge, 2018).

[24] W. Liptay, Ber. Bunsen-Ges. Phys. Chem. 80, 207 (1976).

[25] B. Friedrich, A. Slenczka, and D. Herschbach, Can. J. Phys. 72, 897 (1994).

[26] A. Slenczka, Chem. Eur. J. 5, 1136 (1999).

[27] B. Friedrich and D. Herschbach, J. Chem. Phys. 111, 6157 (1999).

[28] L. Cai, J. Marango, and B. Friedrich, Phys. Rev. Lett. 86, 775 (2001).

[29] H. Sakai, S. Minemoto, H. Nanjo, H. Tanji, and T. Suzuki, Phys. Rev. Lett. 90, 083001 (2003).

[30] B. Friedrich, N. H. Nahler, and U. Buck, J. Mod. Opt. 50, 2677 (2003).

[31] J. H. Nielsen, H. Stapelfeldt, J. Küpper, B. Friedrich, J. J. Omiste, and R. González-Férez, Phys. Rev. Lett. 108, 193001 (2012).

[32] B. Schmidt and B. Friedrich, Front. Phys. 2, 1 (2014).

[33] B. Schmidt and B. Friedrich, Phys. Rev. A 91, 022111 (2015).

[34] C. Z. Bisgaard, O. J. Clarkin, G. Wu, A. M. D. Lee, O. Gessner, C. C. Hayden, and A. Stolow, Science 323, 1464 (2009).
[35] L. Holmegaard, J. L. Hansen, L. Kalhøj, S. Louise Kragh, H. Stapelfeldt, F. Filsinger, J. Küpper, G. Meijer, D. Dimitrovski, M. Abu-samha, C. P. J. Martiny, and L. Bojer Madsen, Nat. Phys. 6, 428 (2010).

[36] J. L. Hansen, H. Stapelfeldt, D. Dimitrovski, M. Abu-samha, C. P. J. Martiny, and L. B. Madsen, Phys. Rev. Lett. 106, 073001 (2011).

[37] A. Landers, T. Weber, I. Ali, A. Cassimi, M. Hattass, O. Jagutzki, A. Nauert, T. Osipov, A. Staudte, M. H. Prior, H. SchmidtBöcking, C. L. Cocke, and R. Dörner, Phys. Rev. Lett. 87, 013002 (2001).

[38] J. Itatani, J. Levesque, D. Zeidler, H. Niikura, H. Pépin, J. C. Kieffer, P. B. Corkum, and D. M. Villeneuve, Nature (London) 432, 867 (2004).

[39] C. Jin, J. B. Bertrand, R. R. Lucchese, H. J. Wörner, P. B. Corkum, D. M. Villeneuve, A.-T. Le, and C. D. Lin, Phys. Rev. A 85, 013405 (2012).

[40] K.-J. Yuan and A. D. Bandrauk, Phys. Rev. A 80, 053404 (2009).

[41] O. Smirnova, Y. Mairesse, S. Patchkovskii, N. Dudovich, D. Villeneuve, P. Corkum, and M. Y. Ivanov, Nature (London) 460, 972 (2009).

[42] A. Rupenyan, J. B. Bertrand, D. M. Villeneuve, and H. J. Wörner, Phys. Rev. Lett. 108, 033903 (2012).

[43] M. A. Baranov, M. Dalmonte, G. Pupillo, and P. Zoller, Chem. Rev. 112, 5012 (2012).

[44] S. R. Manmana, E. M. Stoudenmire, K. R. A. Hazzard, A. M. Rey, and A. V. Gorshkov, Phys. Rev. B 87, 081106(R) (2013).

[45] D. DeMille, Phys. Rev. Lett. 88, 067901 (2002).

[46] B. Schneider, C. Gollub, K.-L. Kompa, and R. de Vivie-Riedle, Chem. Phys. 338, 291 (2007).

[47] B. M. R. Korff, U. Troppmann, K.-L. Kompa, and R. de VivieRiedle, J. Chem. Phys. 123, 244509 (2005).

[48] U. Troppmann, C. M. Tesch, and R. de Vivie-Riedle, Chem. Phys. Lett. 378, 273 (2003).

[49] Q. Wei, S. Kais, B. Friedrich, and D. Herschbach, J. Chem. Phys. 134, 124107 (2011).

[50] Q. Wei, S. Kais, B. Friedrich, and D. Herschbach, J. Chem. Phys. 135, 154102 (2011).

[51] M. Karra, K. Sharma, B. Friedrich, S. Kais, and D. Herschbach, J. Chem. Phys. 144, 094301 (2016).

[52] H. Lefebvre-Brion and R. Field, The Spectra and Dynamics of Diatomic Molecules (Elsevier, Amsterdam, 2004).

[53] M. Sato and T. Tanaka, J. Math. Phys. 43, 3484 (2002).

[54] A. de Souza Dutra, Phys. Rev. A 47, R2435 (1993).

[55] A. González-Lopez, N. Kamran, and P. J. Olver, Commun. Math. Phys. 153, 117 (1993).

[56] Closed-form expression, https://en.wikipedia.org/wiki/Closedform_expression, accessed 12 Feb. 2018.

[57] R. A. Leacock and M. J. Padgett, Phys. Rev. D 28, 2491 (1983).

[58] K. G. Geojo, S. S. Ranjani, and A. K. Kapoor, J. Phys. A 36, 4591 (2003).

[59] M. Lemeshko, M. Mustafa, S. Kais, and B. Friedrich, Phys. Rev. A 83, 043415 (2011).

[60] M. Lemeshko, M. Mustafa, S. Kais, and B. Friedrich, New J. Phys. 13, 063036 (2011).

[61] S. Becker, M. Mirahmadi, B. Schmidt, K. Schatz, and B. Friedrich, Eur. Phys. J. D 71, 149 (2017).

[62] A. Krajewska, A. Ushveridze, and Z. Walczak, Mod. Phys. Lett. A 12, 1225 (1997). 
[63] N. Vilenkin and A. Klimyk, Representation of Lie groups and special functions, in Mathematics and Its Applications (Springer, New York, 1995).

[64] M. Sezgin, A. Y. Verdiyev, and Y. A. Verdiyev, J. Math. Phys. 39, 1910 (1998).

[65] B. Schmidt and B. Friedrich, J. Chem. Phys. 140, 064317 (2014).

[66] A. Gangopadhyaya, J. V. Mallow, and C. Rasinariu, Supersymmetric Quantum Mechanics: An Introduction (World Scientific, Singapore, 2011).

[67] S. S. Ranjani, K. G. Geojo, A. K. Kapoor, and P. K. Panigrahi, Mod. Phys. Lett. A 19, 1457 (2004).

[68] S. B. Bank, G. G. Gundersen, and I. Laine, Annales Academiae Scientiarum Fennicae, Series A. I. Mathematica 6, 369 (1981).

[69] A. González-López, N. Kamran, and P. J. Olver, Contemp. Math. 160, 113 (1994).
[70] D. Gómez-Ullate, N. Kamran, and R. Milson, Found. Comput. Math. 13, 615 (2013).

[71] T. Frankel, The Geometry of Physics: An Introduction, 3rd ed. (Cambridge University, Cambridge, England, 2011).

[72] L. V. Ahlfors, Complex Analysis, 2nd ed. (McGraw-Hill, New York, 1966).

[73] W. M. Boothby, An Introduction to Differentiable Manifolds and Riemannian Geometry, 2nd ed. (Academic, New York, 1986).

[74] H. Zoladek, Topol. Methods Nonlinear Anal. 16, 253 (2000).

[75] B. R. King, Beyond the Quartic Equation (Birkhäuser, Boston, 2009).

[76] T. R. Hagedorn, Journal of Algebra 233, 704 (2000).

[77] G. Teschl, Mathematical Methods in Quantum Mechanics, 2nd ed. (American Mathematical Society, Providence, 2014).

[78] D. Gómez-Ullate, N. Kamran, and R. Milson, Inverse Probl. 23, 1915 (2007). 